\title{
A local calculus for nullhomotopic filling Dehn spheres
}

\author{
GenNARO AmENDOLA
}

\begin{abstract}
We provide a local calculus for the presentation of closed 3-manifolds via nullhomotopic filling Dehn spheres. We use it to define an invariant of closed 3-manifolds by applying the state-sum machinery, and we show how to potentially get lower bounds for the Matveev complexity of $\mathbb{P}^{2}$-irreducible closed 3-manifolds. We also describe an efficient and simple algorithm for constructing a nullhomotopic filling Dehn sphere of each closed 3-manifold from any of its one-vertex triangulations.
\end{abstract}

57M27; 57R42

\section{Introduction}

A presentation of a class of topological objects (in our case closed 3-manifolds) is a class of combinatorial objects (in our case nullhomotopic filling Dehn spheres), such that each combinatorial object defines (say "presents") a unique topological object and each topological object is presented by at least one combinatorial object. A (finite) calculus for a presentation is a (finite) set of moves on the combinatorial objects, such that two combinatorial objects present the same topological object if and only if they are related to each other by a finite sequence of moves in the given set.

Presentations and calculuses are fundamental tools for studying 3-manifolds and for constructing invariants, in fact they translate a topological problem into a combinatorial and perhaps simpler one. For instance, an invariant on the class of topological objects can be defined on the class of combinatorial objects, by checking that it is preserved by the moves of the calculus.

For closed 3-manifolds, there are several different types of presentations, eg triangulations, Heegaard diagrams, surgery (on links) and spines. In the present work we concentrate on nullhomotopic filling Dehn spheres, which dually can be thought of as a particular class of cubulations (see, for instance, Aitchison, Matsumoto and Rubinstein [1], Funar [5], Babson and Chan [2]). The fact that nullhomotopic filling Dehn spheres present closed 3-manifolds is already known (see, for instance, MontesinosAmilibia [12] and Vigara [17]). We will provide here a proof of this result by using a very simple and efficient construction which works for every closed 3-manifold. 
Such a construction is already known and studied (see, for instance, Shtan' ko and Shtogrin [15], Dolbilin and Shtan' ko and Shtogrin [4] and Funar [5]), but we have not found any application to nullhomotopic filling Dehn spheres in the literature.

The main result of this paper is a finite calculus for this presentation. We deduce it from another one, described by Vigara [19], which has been derived from the more general Homma-Nagase calculus [7; 8] (see also Hass and Hughes [6] and Roseman [14]). The most important feature of our calculus is that it is local (ie in order to apply a move, it is enough to look only at the portion of the nullhomotopic filling Dehn sphere involved in the move). In contrast, Vigara's calculus is very interesting and natural, but it has the drawback of not being local; hence, it is not useful for applying the state-sum machinery to define an invariant analogous to the Turaev-Viro one [16].

We will apply the state-sum machinery to our calculus and define an invariant of closed 3-manifolds. Specifically, we will first define the state sum (a polynomial) for a nullhomotopic filling Dehn sphere. Then, we will study how it changes when a move of our calculus is applied and prove that the difference between the state sums of two nullhomotopic filling Dehn spheres of the same closed 3-manifold is an element of a particular ideal of the polynomial ring. (It is at this point that we will use the fact that our calculus is local, because in such a case the alteration due to the moves can be understood and computed explicitly.) Finally, we will get an invariant by taking the coset (with respect to the ideal) represented by the state sum. Some other similar invariants will be also outlined.

As a potential application of this invariant, we will show how to get lower bounds for the Matveev complexity [10] of $\mathbb{P}^{2}$-irreducible closed 3-manifolds in terms of the invariant. The Matveev complexity is usually difficult to compute. Only upper bounds are easy to find (and, typically, they are very precise), while lower bounds are much more difficult to achieve.

\section{Nullhomotopic filling Dehn spheres}

Throughout this paper, all 3-manifolds are assumed to be connected. We will mainly deal with closed 3-manifolds; so $M$ will always denote such a (connected) closed 3 -manifold. Using the Hauptvermutung, we will freely intermingle the differentiable, piecewise linear and topological viewpoints.

Dehn surfaces A subset $\Sigma$ of $M$ is a Dehn surface of $M$ [13] if there exists an abstract closed surface $S$ and a transverse immersion $f: S \rightarrow M$ such that $\Sigma=f(S)$. 
If $f: S \rightarrow M$ is a transverse immersion, the number of preimages of a point of the Dehn surface $\Sigma=f(S)$ is 1, 2 or 3; so there are three types of points in $\Sigma$, depending on this number; they are called simple, double or triple, respectively. Note that the definition of the type of a point of $\Sigma$ does not depend on the particular transverse immersion $f$ we have chosen. In fact, the type of a point can be also defined by looking at a regular neighbourhood (in $M$ ) of the point, as shown in Figure 1. The set

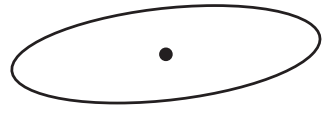

Simple point

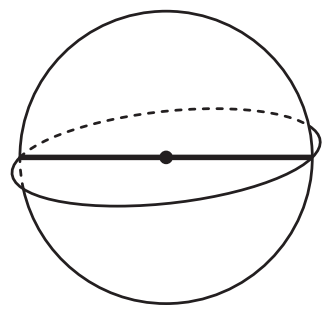

Double point

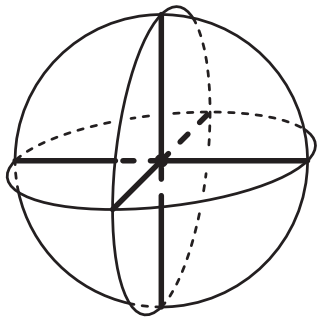

Triple point

Figure 1: Neighbourhoods of points (marked by thick dots) of a Dehn surface

of triple points is denoted by $T(\Sigma)$; nonsimple points are called singular and their set is denoted by $S(\Sigma)$. From now on, in all figures, triple points are always marked by thick dots and the singular set is also drawn thick.

Remark 1 If $f: S \rightarrow M$ and $f^{\prime}: S^{\prime} \rightarrow M$ are transverse immersions such that $f(S)=f^{\prime}\left(S^{\prime}\right)=\Sigma$, then the abstract surfaces $S$ and $S^{\prime}$ are homeomorphic.

Filling Dehn surfaces and cubulations A Dehn surface $\Sigma$ of $M$ is called filling [12] if its singularities induce a cell-decomposition of $M$; more precisely,

- $T(\Sigma) \neq \varnothing$,

- $S(\Sigma) \backslash T(\Sigma)$ consists of intervals (called edges),

- $\Sigma \backslash S(\Sigma)$ consists of discs (called regions),

- $M \backslash \Sigma$ consists of balls.

A cubulation of $M$ is a cell-decomposition of $M$ such that

- each 2-cell (called a face) is glued along 4 edges,

- each 3-cell (called a cube) is glued along 6 faces arranged like the boundary of a cube.

Note that self-adjacencies and multiple adjacencies are allowed. 
The following construction is well-known (see Aitchison, Matsumoto and Rubinstein [1], Funar [5] and Babson and Chan [2], for instance). Let $\mathcal{C}$ be a cubulation of a closed 3-manifold. Consider, for each cube of $\mathcal{C}$, the three squares shown in Figure 2. Then, the subset of $M$ obtained by gluing together all these squares is a filling Dehn surface $\Sigma$ of $M$. Conversely, a cubulation $\mathcal{C}$ can be constructed from a filling Dehn surface $\Sigma$

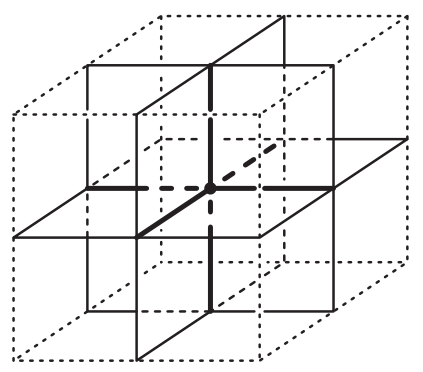

Figure 2: Local behaviour of duality

of $M$ by considering an abstract cube for each triple point of $\Sigma$ and by gluing the cubes together along the faces (the identification of each pair of faces is chosen by following the four germs of regions adjacent to the respective edge of $\Sigma$ ). The cubulation and the filling Dehn surface constructed in such a way are said to be dual to each other.

Nullhomotopic filling Dehn spheres Let $\Sigma$ be a Dehn surface such that $\Sigma=f(S)$, where $f: S \rightarrow M$ is a transverse immersion. If $S$ is a sphere, we will call $\Sigma$ a Dehn sphere (this definition makes sense by Remark 1). A Dehn sphere $\Sigma$ is said to be nullhomotopic if $f$ is homotopic to a constant map (also this definition makes sense, because it does not depend on the particular map $f$ chosen).

In what follows, we will only deal with nullhomotopic filling Dehn spheres. They are enough to study closed 3-manifolds, since they present closed 3-manifolds.

Proposition 2 - Each closed 3-manifold has a nullhomotopic filling Dehn sphere.

- If $\Sigma_{1}$ and $\Sigma_{2}$ are homeomorphic nullhomotopic filling Dehn spheres of closed 3manifolds $M_{1}$ and $M_{2}$ respectively, then $M_{1}$ and $M_{2}$ are also homeomorphic.

Proof We start by proving the first point. Let $\mathcal{T}$ be a one-vertex triangulation of a closed 3-manifold $M$ (all closed 3-manifolds have one-vertex triangulations, as shown in [11], for instance). Consider, for each tetrahedron of $\mathcal{T}$, the four triangles shown in Figure 3. The subset of $M$ obtained by gluing together all these triangles is a filling Dehn surface of $M$; it is actually a nullhomotopic filling Dehn sphere of $M$, 


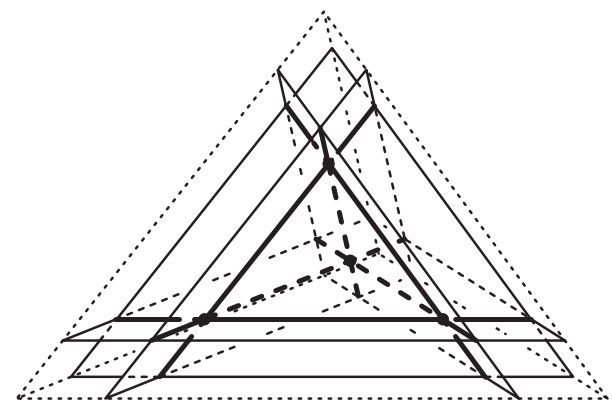

Figure 3: Construction of a nullhomotopic filling Dehn sphere from a onevertex triangulation of a closed 3-manifold

because it can be obtained by starting with a small sphere whose centre is the only vertex of $\mathcal{T}$ and then inflating it.

We do not give a complete proof of the second point because it is essentially the same as that of Casler for standard spines [3]. The idea of the proof is the following. Let $\mathcal{C}_{i}$ be the cubulation of $M_{i}$ dual to $\Sigma_{i}$, for $i=1,2$. The cubulations $\mathcal{C}_{i}$ are defined unambiguously, because the cubes dual to the triple points and the face identifications of them are defined unambiguously (up to homeomorphism) from the Dehn surfaces $\Sigma_{i}$. Since $\Sigma_{1}$ and $\Sigma_{2}$ are homeomorphic, the cubulations $\mathcal{C}_{1}$ and $\mathcal{C}_{2}$ turn out to be isomorphic and hence $M_{1}$ and $M_{2}$ are homeomorphic.

The construction described in the first part of the proof above is the dual counterpart of the well-known construction consisting of dividing a tetrahedron into four cubes [15; $4 ; 5]$.

\section{The calculus}

Throughout this section, $\Sigma$ will always denote a nullhomotopic filling Dehn sphere of a closed 3-manifold $M$.

\subsection{Vigara's calculus}

In [19] Vigara described a finite calculus with three moves (the complete proof was given in [18]).

Theorem 3 (Vigara) Let $\Sigma_{1}$ and $\Sigma_{2}$ be nullhomotopic filling Dehn spheres of closed 3-manifolds $M_{1}$ and $M_{2}$, respectively. Then, $M_{1}$ and $M_{2}$ are homeomorphic if and only if $\Sigma_{1}$ and $\Sigma_{2}$ can be obtained from each other via a sequence of $\operatorname{Vig}_{1}-, \operatorname{Vig}_{2}-$ and $\operatorname{Vig}_{3}-$ moves. 
Let us describe Vigara's moves in detail.

Vig $_{1}$-move The first move is shown in Figure 4 and is called a $\operatorname{Vig}_{1}-$ move (in [19] it is called a finger move 2). It will be called positive if it increases (by four) the number
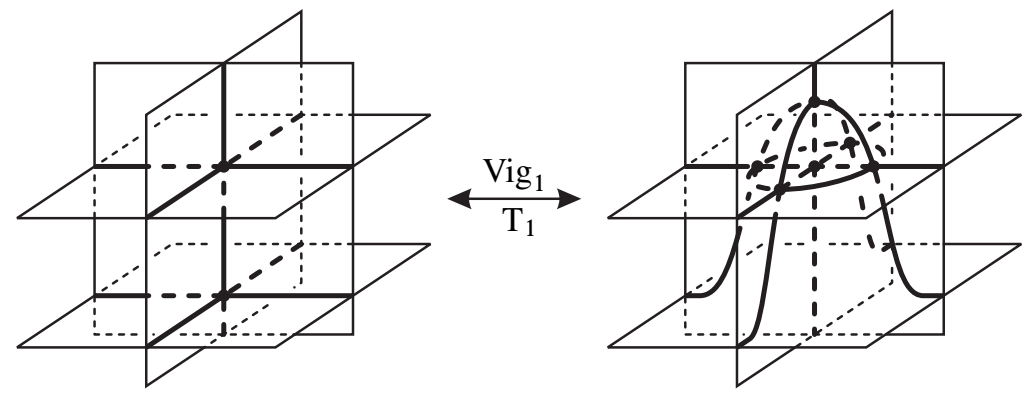

Figure 4: $\operatorname{Vig}_{1}-$ move, also called $\mathrm{T}_{1}-$ move

of triple points of $\Sigma$, and negative otherwise. Note that, if we apply a $\mathrm{Vig}_{1}-$ move to $\Sigma$, the result will be another nullhomotopic filling Dehn sphere of $M$.

Vig $_{2}$-move The second move is shown in Figure 5 and is called a $\operatorname{Vig}_{2}-$ move (in [19] it is called a finger move 1 ). As above, we have positive and negative $\operatorname{Vig}_{2}$-moves,
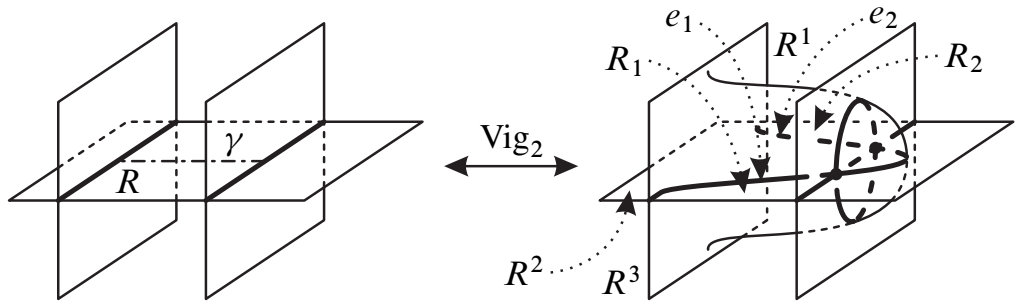

Figure 5: $\operatorname{Vig}_{2}-$ move

depending on whether they increase or decrease (by two) the number of triple points of $\Sigma$. In contrast to the $\operatorname{Vig}_{1}$-move, this move is not local, so it must be described with some care. A positive $\mathrm{Vig}_{2}-$ move is determined by an arc $\gamma$ properly embedded in a region $R$ of $\Sigma$. The move acts on $\Sigma$ as in Figure 5, but, to define its effect unambiguously, we must specify which pairs of regions (out of the four "vertical" ones incident to $R$ at the endpoints of $\gamma$ ) will become adjacent to each other after the move. This is achieved by noting that $R$ is a disc, so its regular neighbourhood in $M$ is a product and hence we can choose for $R$ a transverse orientation. Using it, at each endpoint of $\gamma$ we can tell from each other the two "vertical" regions incident to $R$ as 
being an upper and a lower one, and we can stipulate that the two upper regions will become incident after the move (and similarly for the lower ones).

Obviously, a positive $\operatorname{Vig}_{2}$-move leads to a nullhomotopic filling Dehn sphere of $M$. For the negative case the situation is more complicated. A negative $\mathrm{Vig}_{2}-$ move may lead to a nullhomotopic Dehn sphere that is not filling. For instance, if $R_{1}$ and $R_{2}$ are contained in the same region, after the negative $\operatorname{Vig}_{2}-$ move the "region" $R$ would not be a disc. In order to avoid this loss of fillingness, we will call negative $\mathrm{Vig}_{2}$-moves only those preserving fillingness, so a negative $\mathrm{Vig}_{2}-$ move is the inverse of a positive $\mathrm{Vig}_{2}-$ move. With this convention, if we apply a negative $\mathrm{Vig}_{2}-$ move to $\Sigma$, the result will be another nullhomotopic filling Dehn sphere of $M$.

Vig $_{3}$-move The third move is shown in Figure 6 and is called a $\operatorname{Vig}_{3}-$ move (in [19] it is called a saddle move). In contrast to the other two moves, here we cannot distinguish

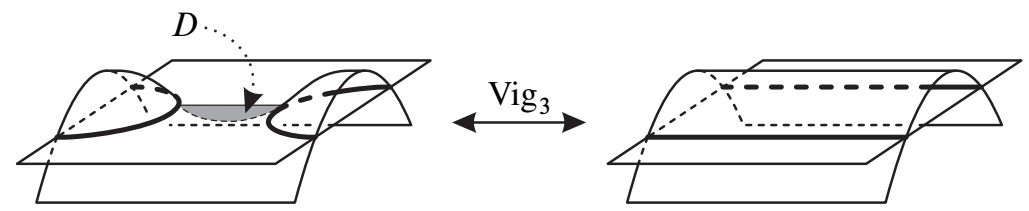

Figure 6: $\operatorname{Vig}_{3}-$ move

between positive and negative $\mathrm{Vig}_{3}$-moves. In fact this move is symmetric. As for the $\mathrm{Vig}_{2}-$ move, this move is not local, so it must be described with some care. A $\mathrm{Vig}_{3}$-move is determined by a disc (say $\Delta$ ) properly embedded in a component (a ball) of $M \backslash \Sigma$, as shown in Figure 6. The move acts on $\Sigma$ as in Figure 6, but, to define its effect unambiguously, we must specify which pairs of regions will unite after the move. This is achieved by the same technique as above, after noting that every region of $\Sigma$ is a disc.

A further restriction is necessary, because a $\operatorname{Vig}_{3}$-move defined in such a way, when applied to $\Sigma$, leads to a nullhomotopic Dehn sphere, which may not be filling. In order to avoid this loss of fillingness, we will call $\mathrm{Vig}_{3}$-moves only those preserving fillingness. With this convention, if we apply a $\operatorname{Vig}_{3}-$ move to $\Sigma$, the result will be another nullhomotopic filling Dehn sphere of $M$.

\subsection{The local calculus}

The $\mathrm{Vig}_{2}-$ and $\mathrm{Vig}_{3}-$ moves of the calculus described above are not useful in defining the invariant, because these moves are not local. In this section we will provide another calculus in which all moves are local. Let us start with the description of the moves. 
$\mathbf{T}_{\mathbf{1}}$-move The first move is the $\operatorname{Vig}_{1}$-move described above (see Figure 4). In order to establish a uniform notation, we call it a $\mathrm{T}_{1}-$ move.

$\mathbf{T}_{2}$-move The second move is shown in Figure 7 and is called a $T_{2}-$ move. In contrast
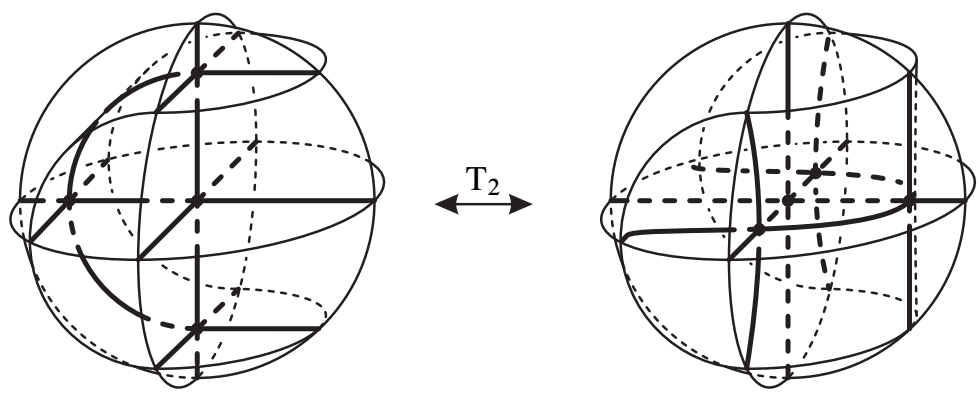

Figure 7: $\mathrm{T}_{2}-$ move

to the $T_{1}$-move, we cannot distinguish between positive and negative $T_{2}-$ moves; in fact this move is symmetric.

$\mathbf{T}_{3}-$ move The third move is shown in Figure 8 and is called a $T_{3}-$ move. It will be
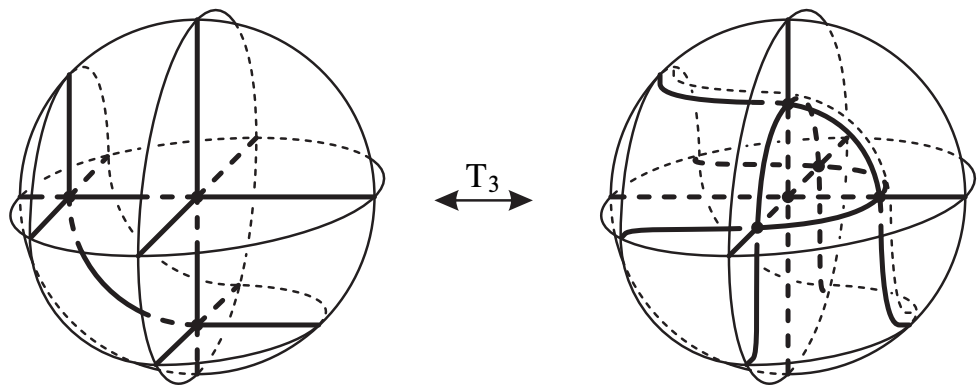

Figure 8: $\mathrm{T}_{3}-$ move

called positive if it increases (by two) the number of triple points of $\Sigma$, and negative otherwise.

$\mathbf{T}_{\mathbf{4}}$-move The fourth move is shown in Figure 9 and is called a $\mathrm{T}_{4}-$ move. As in the case of the $T_{2}$-move, we cannot distinguish between positive and negative $T_{4}$-moves.

B-move The next move is slightly unnatural. It is shown in Figure 10 and is called a B-move. It will be called positive if it increases (by six) the number of triple points of $\Sigma$, and negative otherwise. The configuration shown in Figure 10-right will be called a spiral piping and will be denoted as shown in Figure 10-centre. 

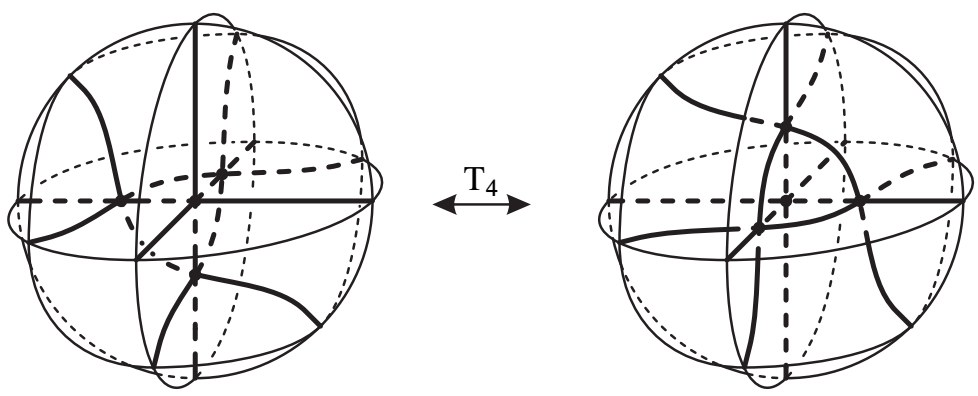

Figure 9: $\mathrm{T}_{4}-$ move
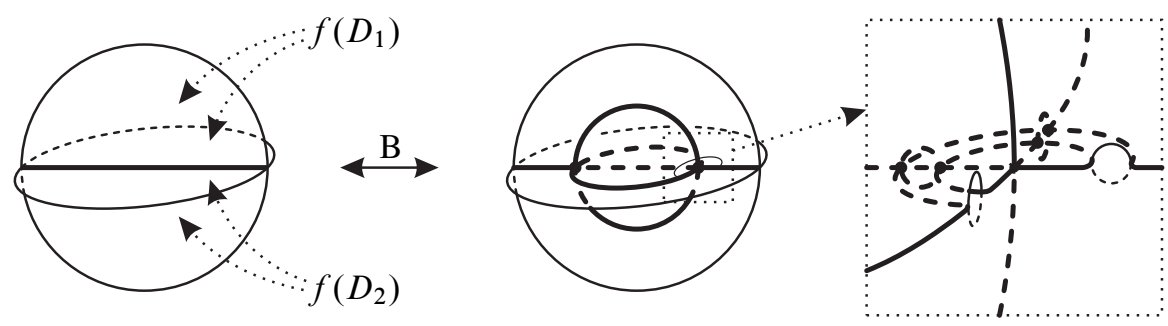

Figure 10: $\mathrm{B}-$ move and spiral piping

S-move The last move is also slightly unnatural. It is shown in Figure 11 and is called an $\mathrm{S}$-move. It is a specialization of the $\mathrm{Vig}_{3}$-move; in fact, it is a $\mathrm{Vig}_{3}$-move applied

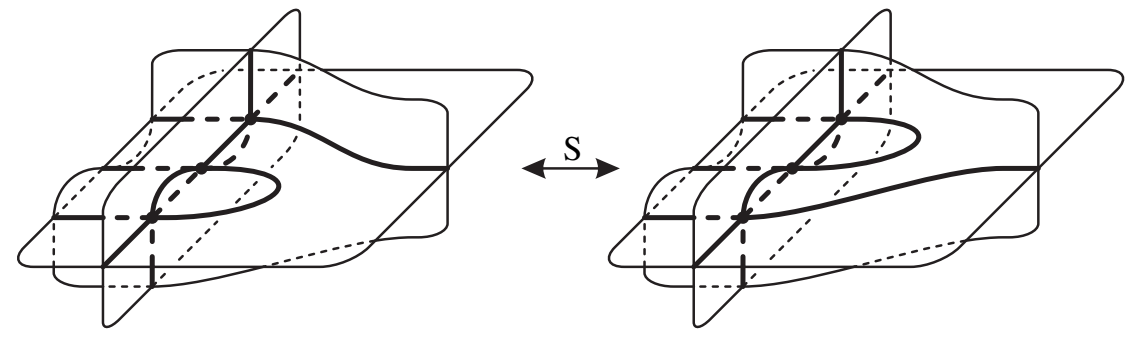

Figure 11: S-move

to a nullhomotopic filling Dehn sphere having a particular shape near the portion involved in the $\mathrm{Vig}_{3}$-move. The $\mathrm{S}$-move is symmetric, so we cannot distinguish between positive and negative $\mathrm{S}$-moves.

Properties of the moves We leave the proof of the following remark to the reader because it is very easy, even if not obvious for the negative B-move case. 
Remark 4 If we apply a $\mathrm{T}_{*}-$, a $\mathrm{B}-$ or an $\mathrm{S}-$ move to a nullhomotopic filling Dehn sphere of a closed 3-manifold $M$, the result will be another nullhomotopic filling Dehn sphere of $M$.

We are now able to state the local calculus.

Theorem 5 Let $\Sigma_{1}$ and $\Sigma_{2}$ be nullhomotopic filling Dehn spheres of closed 3manifolds $M_{1}$ and $M_{2}$, respectively. Then, $M_{1}$ and $M_{2}$ are homeomorphic if and only if $\Sigma_{1}$ and $\Sigma_{2}$ can be obtained from each other via a sequence of $\mathrm{T}_{*}-$, B-and $\mathrm{S}-$ moves.

\subsection{Proof of the calculus}

This section is devoted to the proof of Theorem 5.

By virtue of Remark 4, we need only prove the "only if" part of Theorem 5. By Theorem 3, we have that each pair of nullhomotopic filling Dehn spheres of the same closed 3-manifold can be related by a sequence of $\mathrm{Vig}_{*}-$ moves. Hence it is enough to prove that each $\mathrm{Vig}_{*}-$ move is a composition of $\mathrm{T}_{*}-, \mathrm{B}-$ and $\mathrm{S}-$ moves. For the $\mathrm{Vig}_{1}$-move there is nothing to prove, because each $\mathrm{Vig}_{1}$-move is already a $\mathrm{T}_{1}$-move. With the following lemma we analyse the $\mathrm{Vig}_{2}-$ move.

Lemma 6 Each $\mathrm{Vig}_{2}-$ move is a composition of $\mathrm{T}_{*}$-and $\mathrm{B}$-moves.

Proof Obviously, it is enough to prove the statement only for positive $\mathrm{Vig}_{2}$-moves. Therefore, we consider a positive $\operatorname{Vig}_{2}$-move between two nullhomotopic filling Dehn spheres (say $\Sigma$ and $\Sigma^{\prime}$ ); see Figure 5. Let us first suppose that the closure in $\Sigma^{\prime}$ of one of the two regions $R_{1}$ and $R_{2}$ is a closed disc incident to at least three triple points; see Figure 12 for example. In such a case the sequence of $T_{*}$-moves shown in Figure 13 is equivalent to the $\mathrm{Vig}_{2}$-move. For the sake of simplicity, we have shown only the singular set contained in the "horizontal plane" of Figure 12, but we invite the reader to figure out the 3-dimensional picture. We have shown the moves for a particular case only; in the general one, we have a positive $\mathrm{T}_{1}$-move, a negative $\mathrm{T}_{3}-$ move, and then pairs of a positive and a negative $\mathrm{T}_{1}$-move (depending on the number of triple points adjacent to the region).

Suppose now that the closure of both $R_{1}$ and $R_{2}$ is not a disc, but at least one of them, say $R_{1}$ (the case of $R_{2}$ being symmetric), is incident to not less than three triple points. We can repeat the procedure above unless $R_{1}$ is incident to the edge $e_{1}$ more than once (see Figure 5). In such a case, however, we can repeat the procedure, but, when 


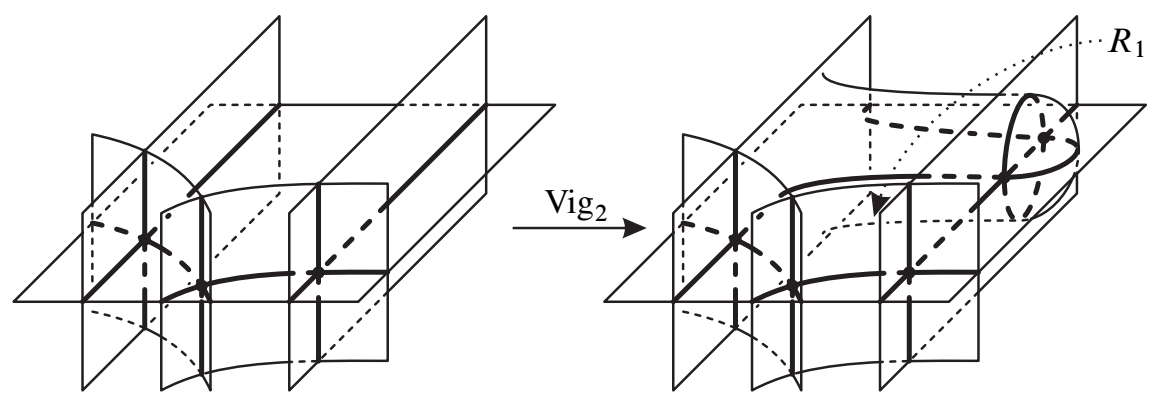

Figure 12: A positive $\operatorname{Vig}_{2}-$ move when the closure in $\Sigma^{\prime}$ of $R_{1}$ is a closed disc incident to at least three triple points (the case of $R_{2}$ being symmetric).

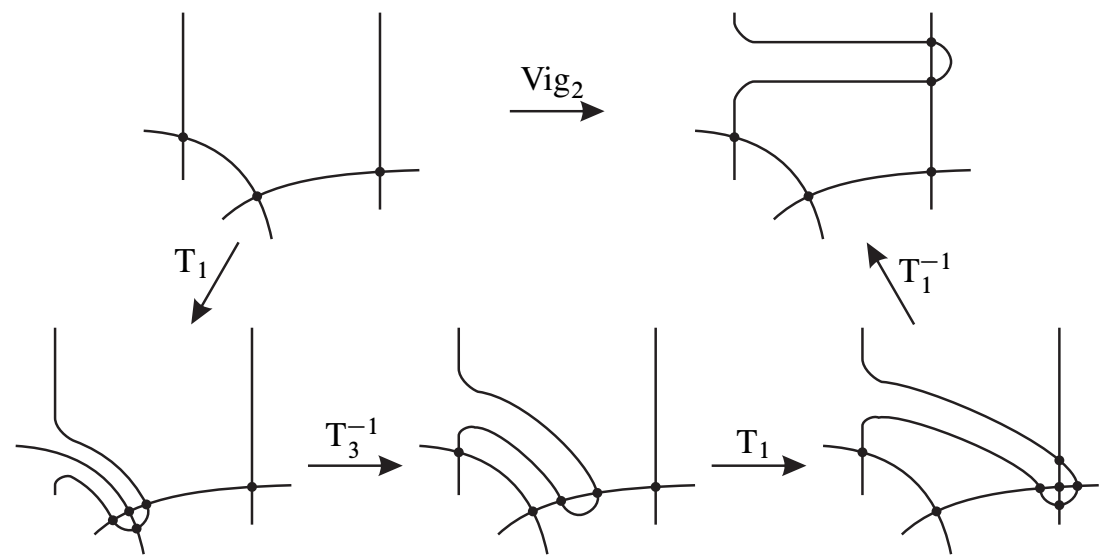

Figure 13: The sequence of $\mathrm{T}_{*}$-moves equivalent to the positive $\mathrm{Vig}_{2}-$ move shown in Figure 12

we need to pass along the edge $e_{1}$, we should add two $\mathrm{Vig}_{2}-$ moves (a positive and a negative one). More precisely, $R_{1}$ may be equal to $R^{i}$ for $i=1,2,3$ (see Figure 5). We have shown in Figure 14 the moves that are performed if $R_{1}=R^{2}$, the other two cases being analogous. Note that the two $\mathrm{Vig}_{2}-$ moves we have added are compositions of $\mathrm{T}_{*}$-moves because the closure of the region $R^{\prime}$ is a closed disc incident to three triple points (see Figure 14).

Finally, suppose both $R_{1}$ and $R_{2}$ are incident to at most two triple points. In such a case, we can suitably apply a positive $\mathrm{B}-$ move near the boundary of $R_{1}$ so that it comes to be incident to at least three triple points. Then, we can apply the $\mathrm{T}_{*}-$ moves described above and conclude by applying a negative $\mathrm{B}-$ move. Therefore, we have proved that each $\mathrm{Vig}_{2}-$ move is a composition of $\mathrm{T}_{*}-$ and $\mathrm{B}-$ moves. 


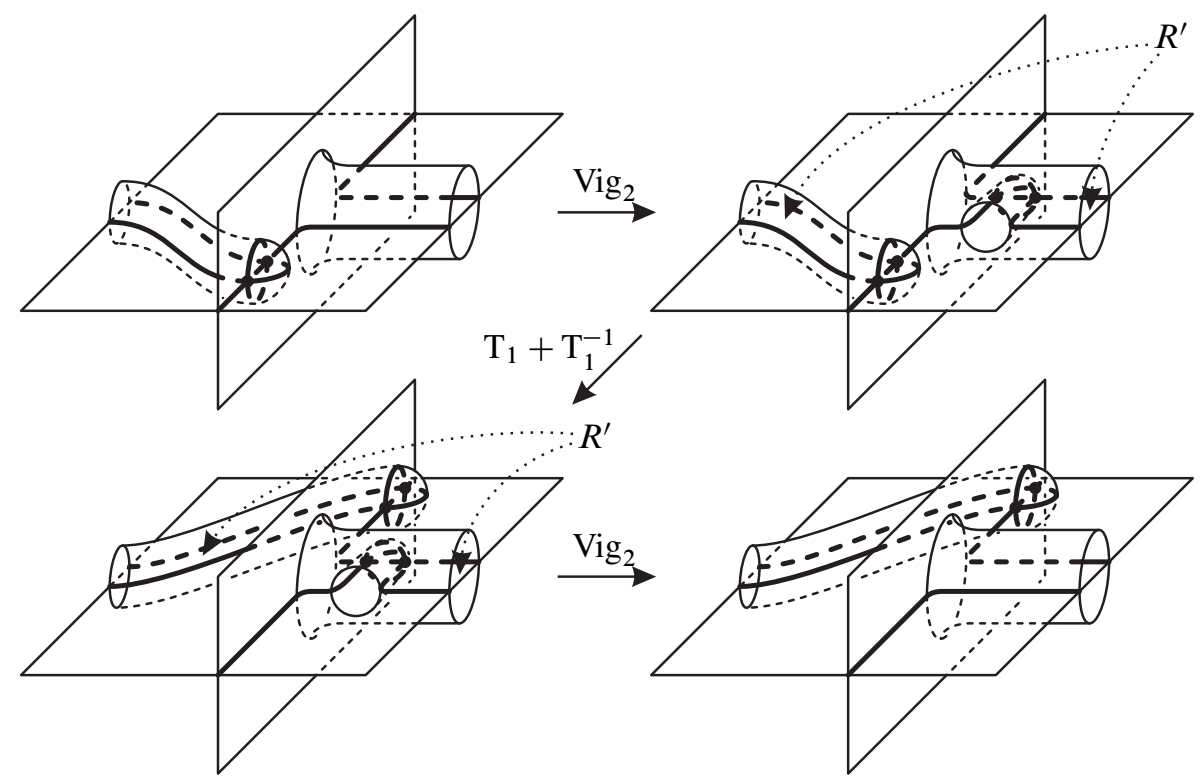

Figure 14: If $R_{1}$ is incident more than once to the edge $e_{1}$, we must slightly modify the procedure.

Before analysing the $\mathrm{Vig}_{3}-$ move, we introduce some notation and state two technical lemmas.

Passing through spiral pipings The move shown in Figure 15 is called a spiral piping passing move. It will be called positive if it increases (by four) the number of triple
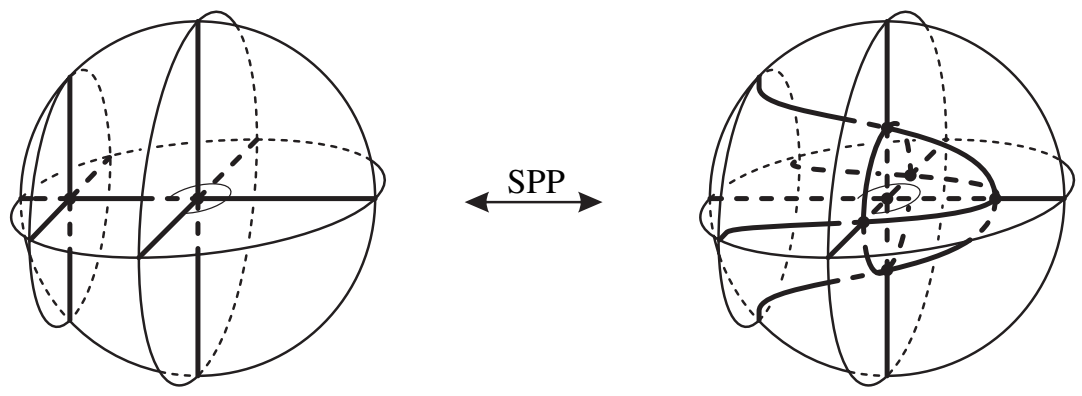

Figure 15: Spiral piping passing move

points of the Dehn sphere, and negative otherwise. It is worth noting that this move is only a particular case of the piping passing move of [19].

Lemma 7 Each spiral piping passing move is a composition of $\mathrm{T}_{*}-$ moves. 
Proof Obviously, it is enough to prove the statement only for positive spiral piping passing moves. The sequence of $T_{*}$-moves shown in Figure 16 is equivalent to the positive spiral piping passing move. For the sake of simplicity, we have shown only the
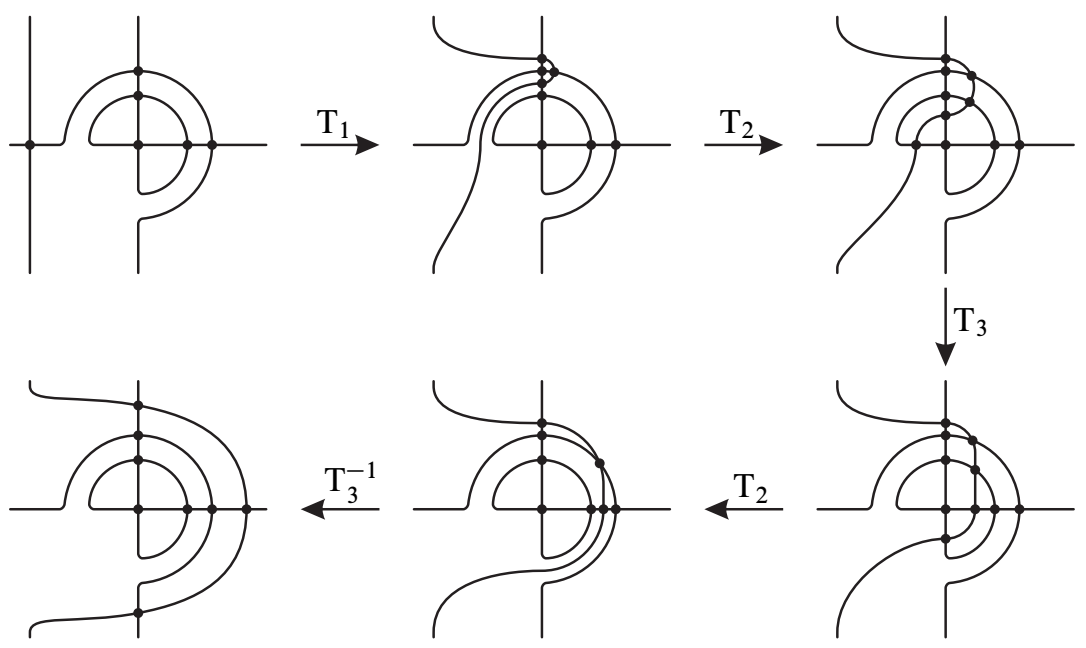

Figure 16: The sequence of $\mathrm{T}_{*}$-moves equivalent to the positive spiral piping passing move

singular set contained in the "horizontal plane" of Figure 15, but we invite the reader to figure out the 3-dimensional picture.

The wall In the proof we will need a nonlocal move; however, it will turn out to be a composition of $\mathrm{T}_{*}-$ and $\mathrm{B}-$ moves.

Let $\Sigma$ be a nullhomotopic filling Dehn sphere of a closed 3-manifold $M$. Let $D$ be a closed disc embedded in $M$ such that

- $\partial D \subset \Sigma$,

- $\operatorname{int}(D) \cap \Sigma=\varnothing$,

- $\partial D \cap T(\Sigma)=\varnothing$,

- $\#(\partial D \pitchfork S(\Sigma)) \geqslant 2$.

Let $B$ be a small regular neighbourhood of $D$ in $M$. Obviously, $B$ is a ball whose boundary $\partial B$ is a sphere intersecting some edges of $\Sigma$. Let $\Sigma_{D, p}$ be the Dehn surface obtained from $\Sigma \cup \partial B$ by replacing a small neighbourhood of a triple point $p$ (of $\Sigma \cup \partial B$ ) contained in $\partial B$ with a spiral piping as shown in Figure 17. Note that the choice of the triple point $p$ is arbitrary, but the orientation of the spiral piping is not. 


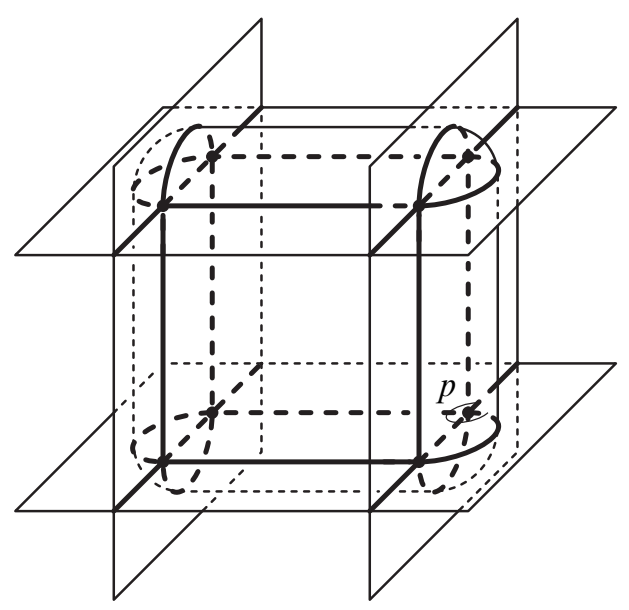

Figure 17: A wall with $\#(\partial D \cap S(\Sigma))=4$

Note also that we can think of $p$ both as a (double) point of $\Sigma$ and as a (triple) point of $\Sigma_{D, p}$. Moreover, $\Sigma_{D, p}$ is a nullhomotopic filling Dehn sphere of $M$.

The configuration shown in Figure 17 is said to be a wall of $\Sigma_{D, p}$ with respect to the triple $(\Sigma, D, p)$, and the move between $\Sigma$ and $\Sigma_{D, p}$ is said to be a positive $\mathrm{W}$-move. Note that this move is similar to the B-move, but it is not local, and actually there are infinitely many different $\mathrm{W}$-moves depending on the number of singular points in $\partial D$. A negative $\mathrm{W}-$ move is the inverse of a positive $\mathrm{W}$-move. Note that, in order to apply a negative $\mathrm{W}$-move to a nullhomotopic filling Dehn sphere of $M$, we should only check that the result (which is anyway a nullhomotopic Dehn sphere of $M$ ) is filling.

We have the following lemma, whose (long and technical) proof will be postponed for a while.

Lemma 8 Each $\mathrm{W}$-move is a composition of $\mathrm{T}_{*}-$ and $\mathrm{B}$-moves.

Proof of the calculus After stating Lemma 8, we conclude the proof of the calculus.

Proof of Theorem 5 By virtue of the discussion above, we need only prove that each $\mathrm{Vig}_{3}-$ move is a composition of $\mathrm{T}_{*}-, \mathrm{B}-$ and $\mathrm{S}$-moves. Let us consider a $\mathrm{Vig}_{3}-$ move between two nullhomotopic filling Dehn spheres (say $\Sigma$ and $\Sigma^{\prime}$ ); see Figure 6 . The idea is to modify the portion of $\Sigma$ involved in the move (shown in Figure 6-left) via some $\mathrm{T}_{*}-$ and $\mathrm{W}$-moves in order to apply an $\mathrm{S}$-move, and then to reconstruct the portion of $\Sigma^{\prime}$ involved in the move (shown in Figure 6-right). Therefore, we start by applying two positive $\mathrm{W}$-moves and two positive $\mathrm{T}_{*}$-moves, as shown in Figure 18 

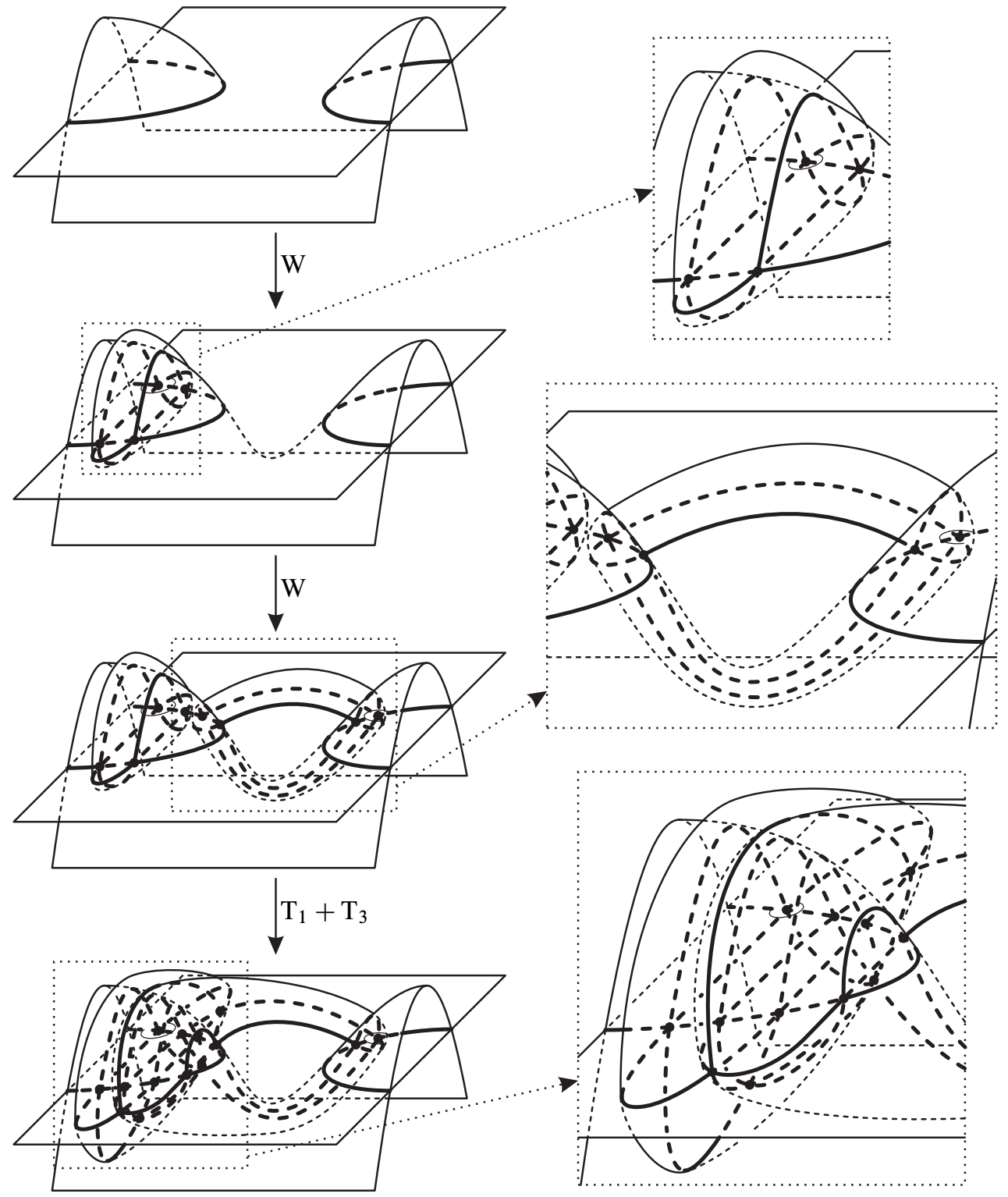

Figure 18: $\mathrm{T}_{*}-, \mathrm{W}-$ and $\mathrm{S}-$ moves equivalent to the $\mathrm{Vig}_{3}-$ move (first part)

(see also Figure 19-left). Then, we can apply an $\mathrm{S}$-move as shown in Figure 19 (where only the singular set contained in the "horizontal plane" of Figure 18-bottom and Figure 20-top is shown). Finally, we apply two negative $\mathrm{T}_{*}-$ moves and two negative $\mathrm{W}$-moves, as shown in Figure 20 (see also Figure 19-right). Note that indeed the 


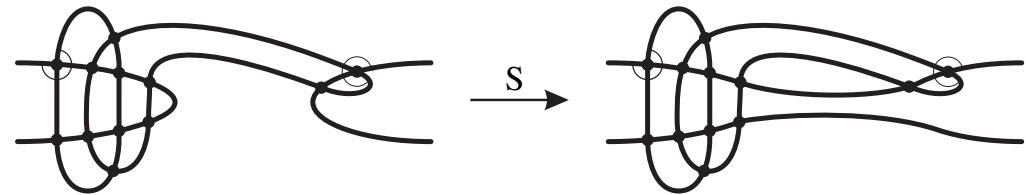

Figure 19: $\mathrm{T}_{*}-, \mathrm{W}-$ and $\mathrm{S}-$ moves equivalent to the $\mathrm{Vig}_{3}-$ move (second part)

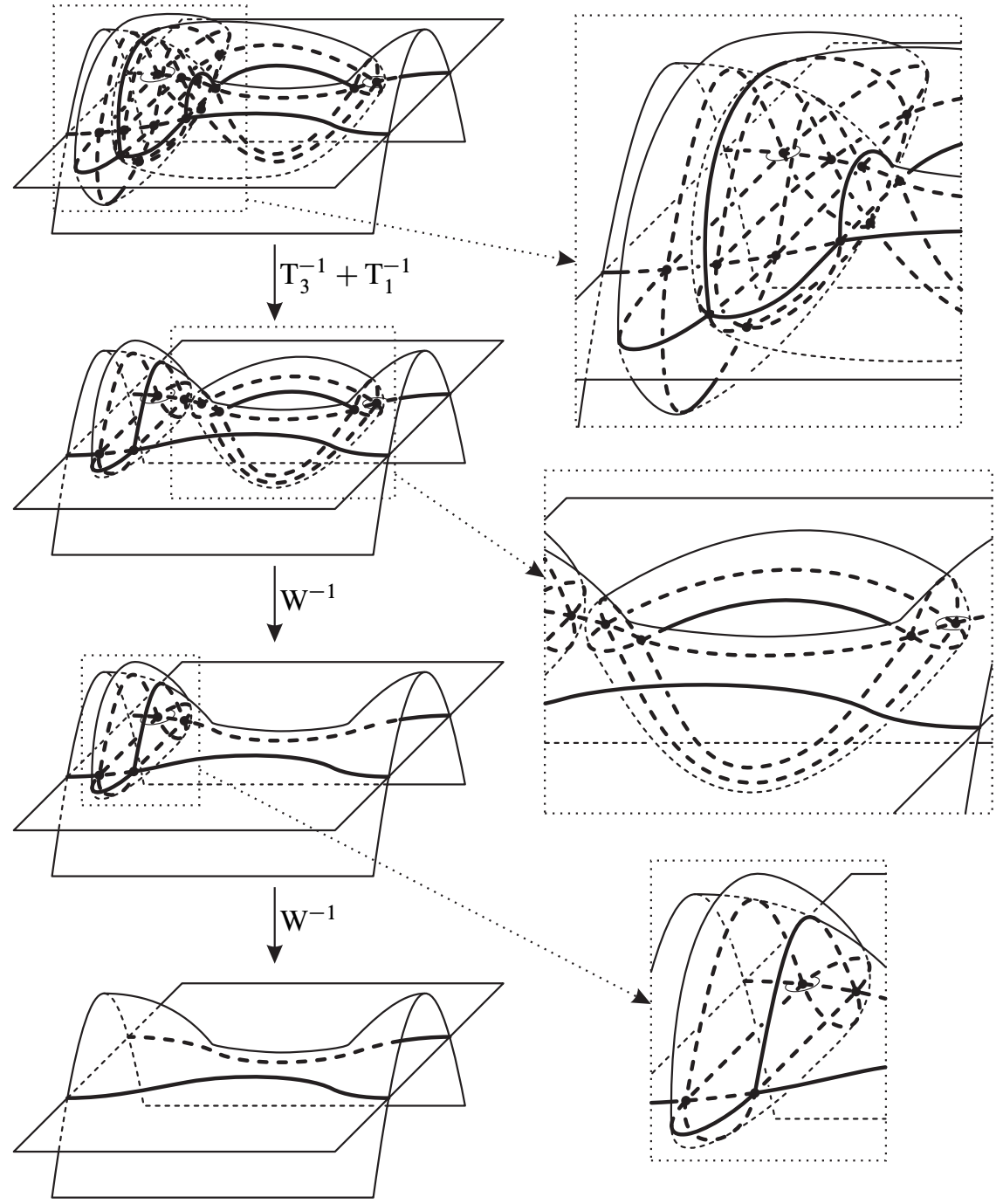

Figure 20: $\mathrm{T}_{*}-, \mathrm{W}-$ and $\mathrm{S}-$ moves equivalent to the $\mathrm{Vig}_{3}-$ move (third part) 
negative $\mathrm{W}$-moves preserve fillingness because the $\mathrm{Vig}_{3}$-move does. We have proved that each $\mathrm{Vig}_{3}-$ move is a composition of $\mathrm{T}_{*}-, \mathrm{W}-$ and $\mathrm{S}$-moves; so, in order to conclude the proof, it is enough to note that each $\mathrm{W}$-move is a composition of $\mathrm{T}_{*}-$ and B-moves by virtue of Lemma 8 .

The very technical proof We conclude this section with the proof of the fact that each $\mathrm{W}$-move is a composition of $\mathrm{T}_{*}-$ and $\mathrm{B}$-moves. We warn the reader that this proof is quite long and technical, so it can be skipped at first reading.

Proof of Lemma 8 Obviously, it is enough to prove that each positive $\mathrm{W}$-move is a composition of $\mathrm{T}_{*}-$ and $\mathrm{B}$-moves. As a matter of fact, it is enough to prove that each positive $\mathrm{W}$-move is a composition of $\mathrm{T}_{*}-\mathrm{B}-$ and $\mathrm{Vig}_{2}-$ moves, because each $\mathrm{Vig}_{2}-$ move is a composition of $\mathrm{T}_{*}-$ and $\mathrm{B}-$ moves by virtue of Lemma 6 . Hence, let us consider a positive $\mathrm{W}$-move between two nullhomotopic filling Dehn spheres $\Sigma$ and $\Sigma_{D, p}$ of a closed 3-manifold $M$, where $D$ is a closed disc embedded in $M$ such that $\partial D \subset \Sigma$, int $(D) \cap \Sigma=\varnothing, \partial D \cap T(\Sigma)=\varnothing, \#(\partial D \pitchfork S(\Sigma)) \geqslant 2$. In the figures below we will draw the case where \# $(\partial D \cap S(\Sigma))=4$ as in Figure 17, the other cases being analogous. Let us consider a small disc $D^{\prime}$ near $p$ as shown in Figure 21-left. We choose $D^{\prime}$ so that $D \cap D^{\prime}$ is the small triangle shown in Figure 21-left. Let $C$ be
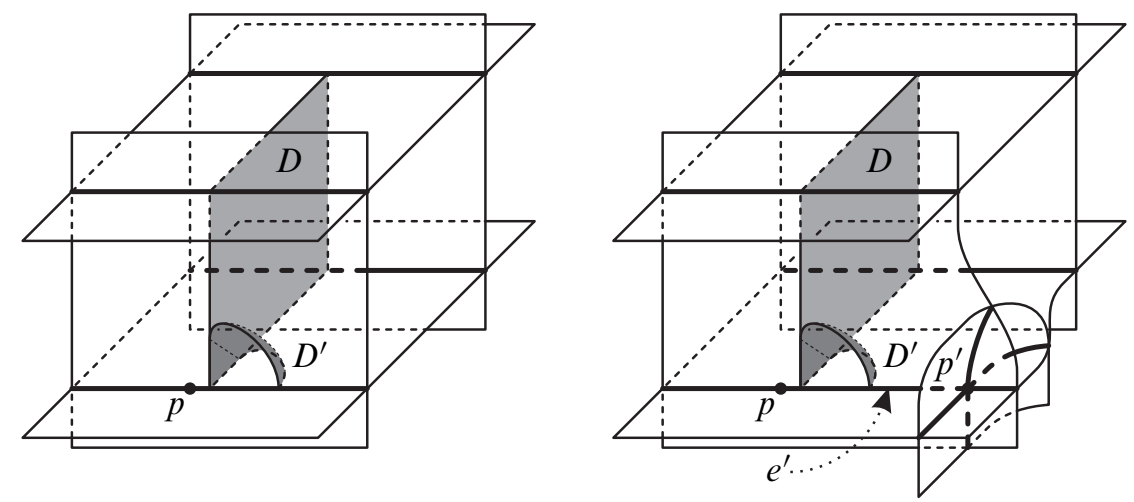

Figure 21: A small disc $D^{\prime}$ near $p$ (left) and the choice of the triple point $p^{\prime}$ (right)

the connected component of $M \backslash \Sigma$ containing $\operatorname{int}(D)$. Since $\Sigma$ is filling, we have that $C$ is a ball and that it is divided by the two discs $D$ and $D^{\prime}$ into three balls, one of which, say $C^{\prime}$, is not incident to the triangle $D \cap D^{\prime}$. The idea of the proof is to create a small wall with respect to the triple $\left(\Sigma, D^{\prime}, p\right)$ and then to move it through the ball $C^{\prime}$. 
Let us start by creating the small wall. Let us call $e$ the edge of $\Sigma$ containing the triple point $p$. It is divided into two parts by the closure of the triangle $D \cap D^{\prime}$; let us call $e^{\prime}$ the one intersecting twice the closure of $D^{\prime}$, and $p^{\prime}$ the triple point at the end of $e^{\prime}$; see Figure 21-right. (Note that $e^{\prime}$ may intersect more than once the closure of $D$, but this does not affect the procedure). In order to get $\Sigma_{D^{\prime}, p}$ from $\Sigma$, we apply the moves shown in Figure 22.
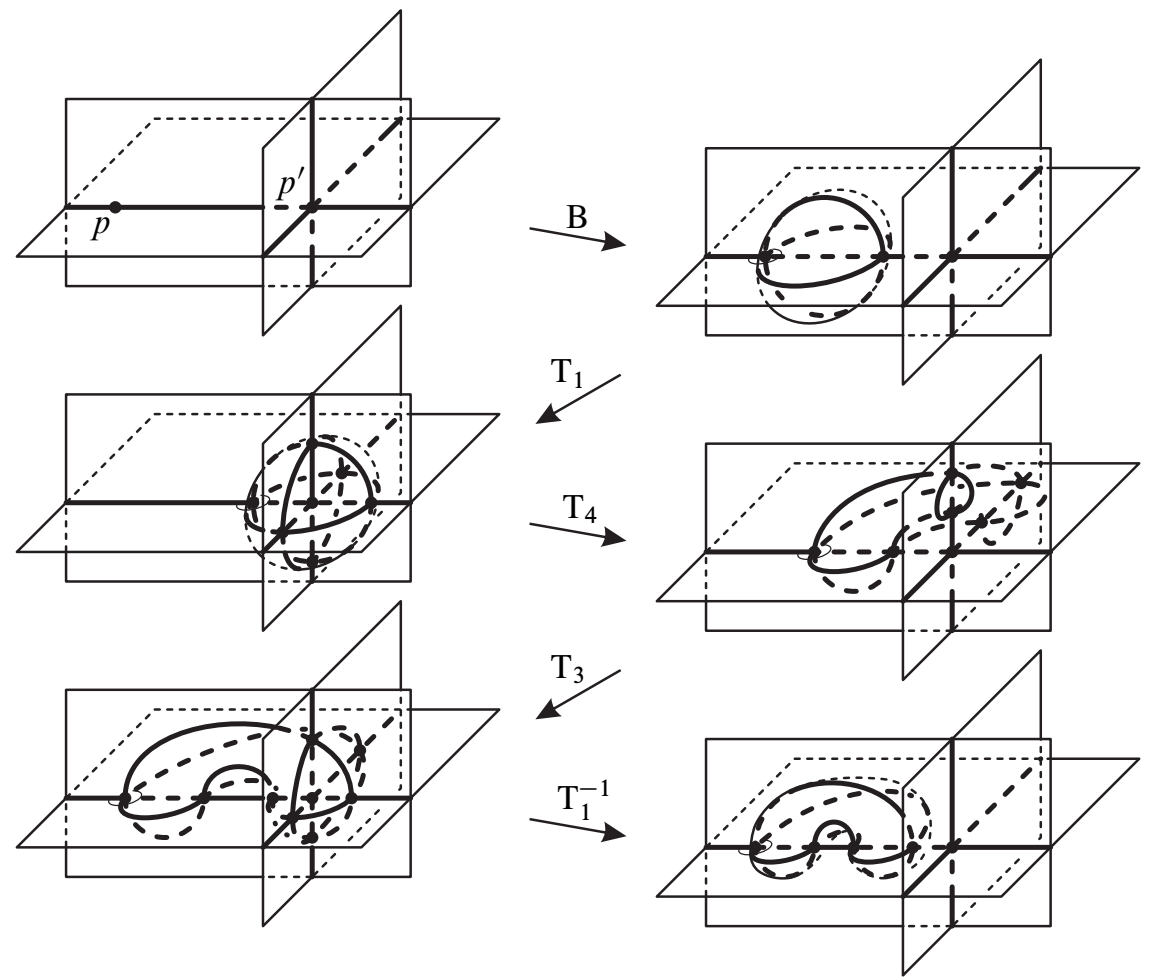

Figure 22: The creation of the small wall

We have created the small wall; we now need to move it through the ball $C^{\prime}$. Note that, if the two Dehn spheres $\Sigma_{D, p}$ and $\Sigma_{D^{\prime}, p}$ are isotopic, we have done; so we suppose they are not isotopic.

Initially, let us suppose that the closure of $C^{\prime}$ is a closed ball. We can move the disc $D^{\prime}$ through the ball $C^{\prime}$ via an isotopy keeping fixed the triangle $D \cap D^{\prime}$ (see Figure 23 for an example). If we consider also the trivalent graph $S(\Sigma) \cap \partial C^{\prime}$, a simple general position argument tells us that the isotopy can be substituted by 1to2-moves and 0to2-moves; see Figure 24. (The disc we are moving is drawn in grey.) 


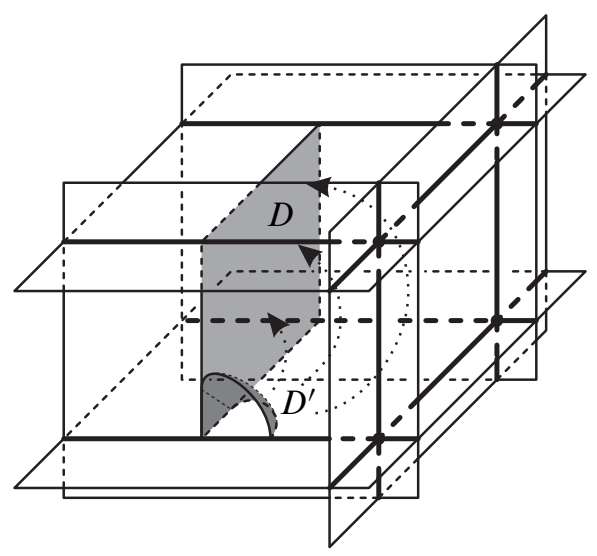

Figure 23: Moving the disc $D^{\prime}$ through $C^{\prime}$
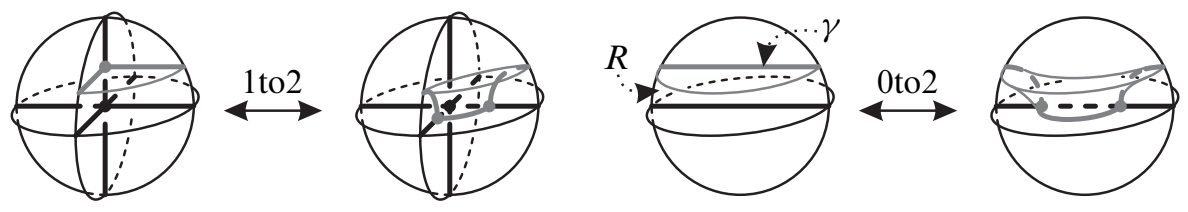

Figure 24: 1to2-move (left) and 0to2-move (right)

We now prove that each 0to2-move is a composition of 1 to $2-$ and $\operatorname{Vig}_{2}$-moves. Consider a 0to2-move (see Figure 24-right). Let $R$ be the connected component of $\Sigma \backslash\left(S(\Sigma) \cup \partial D^{\prime}\right)$ that is divided in two after the 0to2-move, and $\gamma$ the arc of $\partial D^{\prime}$ that is moved after the 0to2-move. Note that the boundary of $C^{\prime}$ appears near the portion of it involved in the move as in Figure 25-left, because $R$ is a disc and the endpoints of $\gamma$ are double points of $\Sigma$. The 1 to $2-$ and $\operatorname{Vig}_{2}$-moves shown in Figure 25 are equivalent to the 0 to $2-$ move.

We have proved that the isotopy of $D^{\prime}$ above can be substituted by 1 to2- and $\mathrm{Vig}_{2}-$ moves. We now consider the small wall. If we substitute each 1to2-move with a $\mathrm{T}_{1}-$ and a $\mathrm{T}_{3}$-move as shown in Figure 26, we obtain a sequence of $\mathrm{T}_{*}-$ and $\mathrm{Vig}_{2}-$ moves moving the wall and transforming $\Sigma_{D^{\prime}, p}$ into $\Sigma_{D, p}$. (Note that indeed the negative $\mathrm{Vig}_{2}$-move preserves fillingness.) Hence, we have proved the statement if the closure of $C^{\prime}$ is a closed ball.

Consider now the general case; namely, we no longer suppose that the closure of $C^{\prime}$ is a closed ball. We need to prove that $\Sigma_{D, p}$ can be obtained from $\Sigma_{D^{\prime}, p}$ via $\mathrm{T}_{*}-$, Band $\mathrm{Vig}_{2}$-moves. The technique is analogous to that used in the case already analysed when the closure of $C^{\prime}$ is a closed ball, but here we must prepare the Dehn sphere 

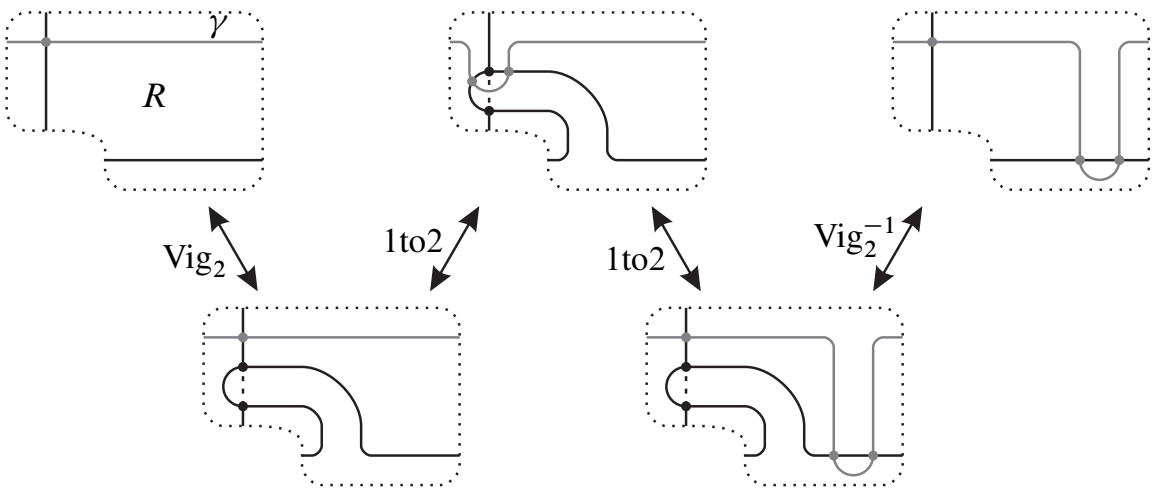

Figure 25: Each 0to2-move is a composition of 1to2- and $\mathrm{Vig}_{2}-$ moves.

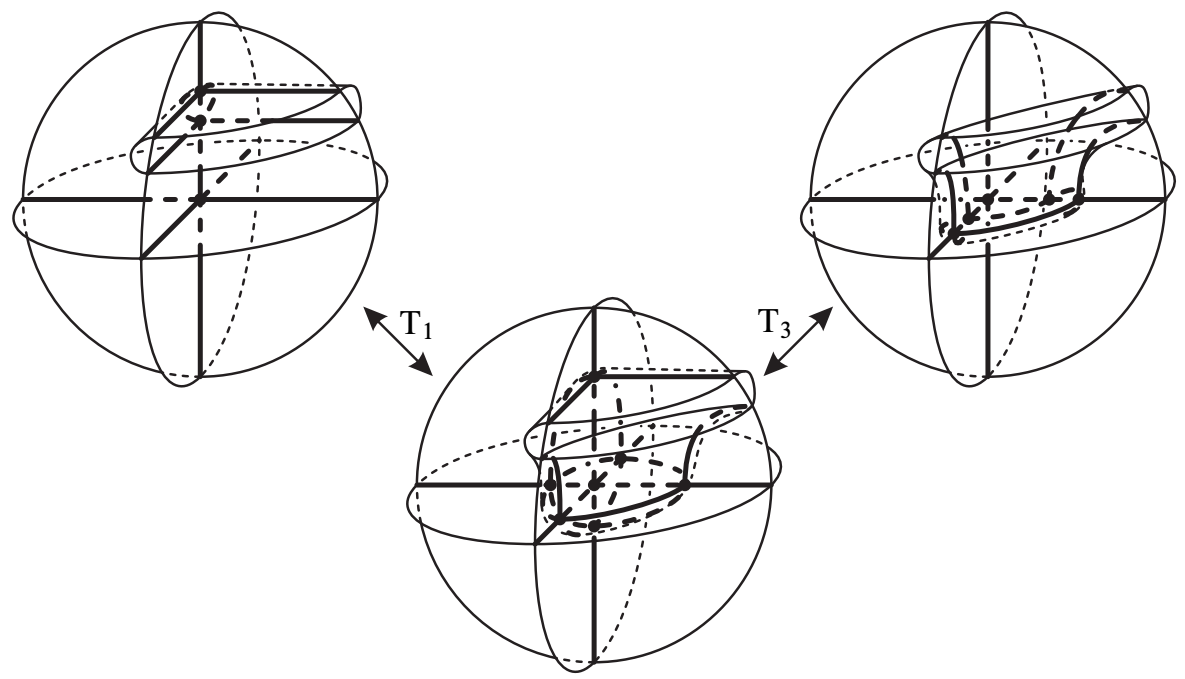

Figure 26: Each 1to2-move is substituted with two $\mathrm{T}_{*}-$ moves.

$\Sigma_{D^{\prime}, p}$ before moving the small wall. For the sake of simplicity, we continue calling $\Sigma_{D^{\prime}, p}$ all Dehn spheres obtained throughout the procedure. For the sake of clarity, in Figures Figure 29, 30, 32 and 33, the wall has been substituted with the disc $D^{\prime \prime}$ : in order to obtain the complete picture, the reader should replace $D^{\prime \prime}$ with the boundary of a small regular neighbourhood of $D^{\prime \prime}$ and add a spiral piping at $p$.

First of all, we apply a positive B-move, a positive spiral piping passing move (which is a composition of $T_{*}$-moves by virtue of Lemma 7) and a $T_{4}$-move; see Figure 27. Then, we apply positive $\mathrm{B}$-moves and positive $\mathrm{Vig}_{2}$-moves near the boundary of $D$, as shown in Figure 28. Afterwards, we apply two positive $\mathrm{T}_{1}-$ and two $\mathrm{T}_{4}-$ moves (the 


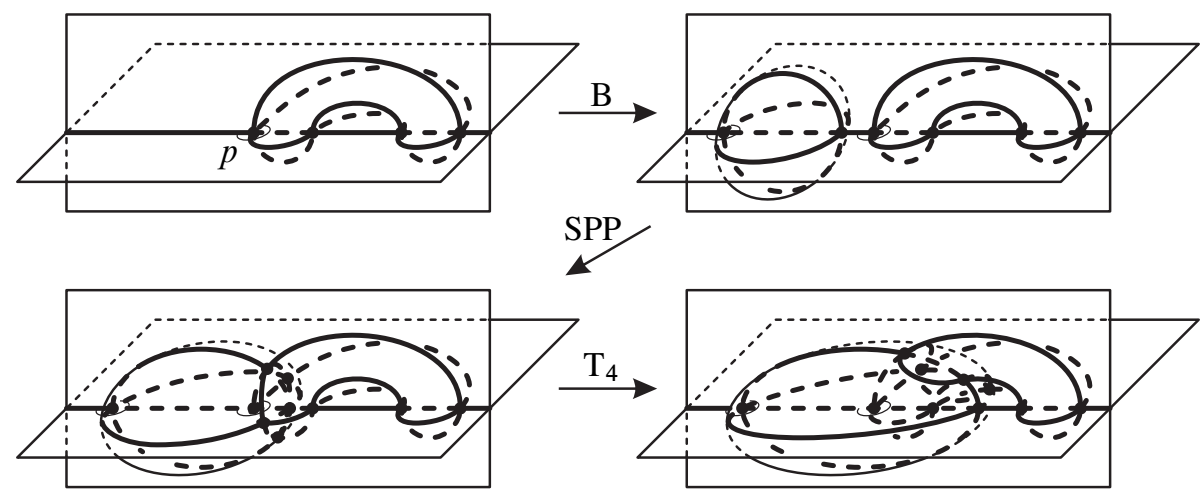

Figure 27: Preparation before moving the small wall (first part)

result is shown in Figure 29). Finally, we apply three pairs of a $T_{1}-$ and a $T_{3}-$ move, as when we have substituted the 1to2-moves (the result is shown in Figure 30). Note that here we have used the hypothesis that $\#(\partial D \cap S(\Sigma)) \geqslant 2$.

Now, we look at the closure of $C^{\prime}$. It can be thought of as an abstract closed ball with some self-identifications on the boundary. In order to simplify such identifications, we apply a B-move and a positive $\mathrm{T}_{1}$-move for each triple point of $\Sigma_{D^{\prime}, p}$ where we have a self-adjacency of the closure of $C^{\prime}$; then, we apply a positive spiral piping passing move or a positive $T_{1}$-move for each edge of $\Sigma_{D^{\prime}, p}$ where we have a self-adjacency of the closure of $C^{\prime}$; see Figure 31. Note that we must avoid the situation where two spiral pipings are on the same edge, for otherwise we can apply neither the positive spiral piping passing move nor the positive $\mathrm{T}_{1}$-move; this can be achieved by considering that, since $S\left(\Sigma_{D^{\prime}, p}\right)$ is a hexavalent graph, we can choose for each triple point an edge adjacent to it so that each edge is chosen for at most one triple point (this holds for each graph containing no connected component that is a tree). Note also that the small wall is not affected by these moves. Now, the self-identifications are along discs, each of which is contained in a region of $\Sigma$ and can be thought of as being as small as we want (with respect to $C^{\prime}$ ).

We can finally move the small wall through the ball $C^{\prime}$, as we have done above in the case when the closure of $C^{\prime}$ is a ball. We must be careful now because the closure of $C^{\prime}$ is not a ball; however, the isotopy of $D^{\prime}$ can be chosen so that $D^{\prime}$ is always incident to one side of each self-adjacency disc at most. With such an isotopy we can repeat the procedure above for the case when the closure of $C^{\prime}$ is a ball. The result is shown in Figure 32.

To conclude, we firstly apply, in reverse order, the moves implemented above to simplify the self-adjacency of the closure of $C^{\prime}$ (see Figure 31). Afterwards, we apply some 

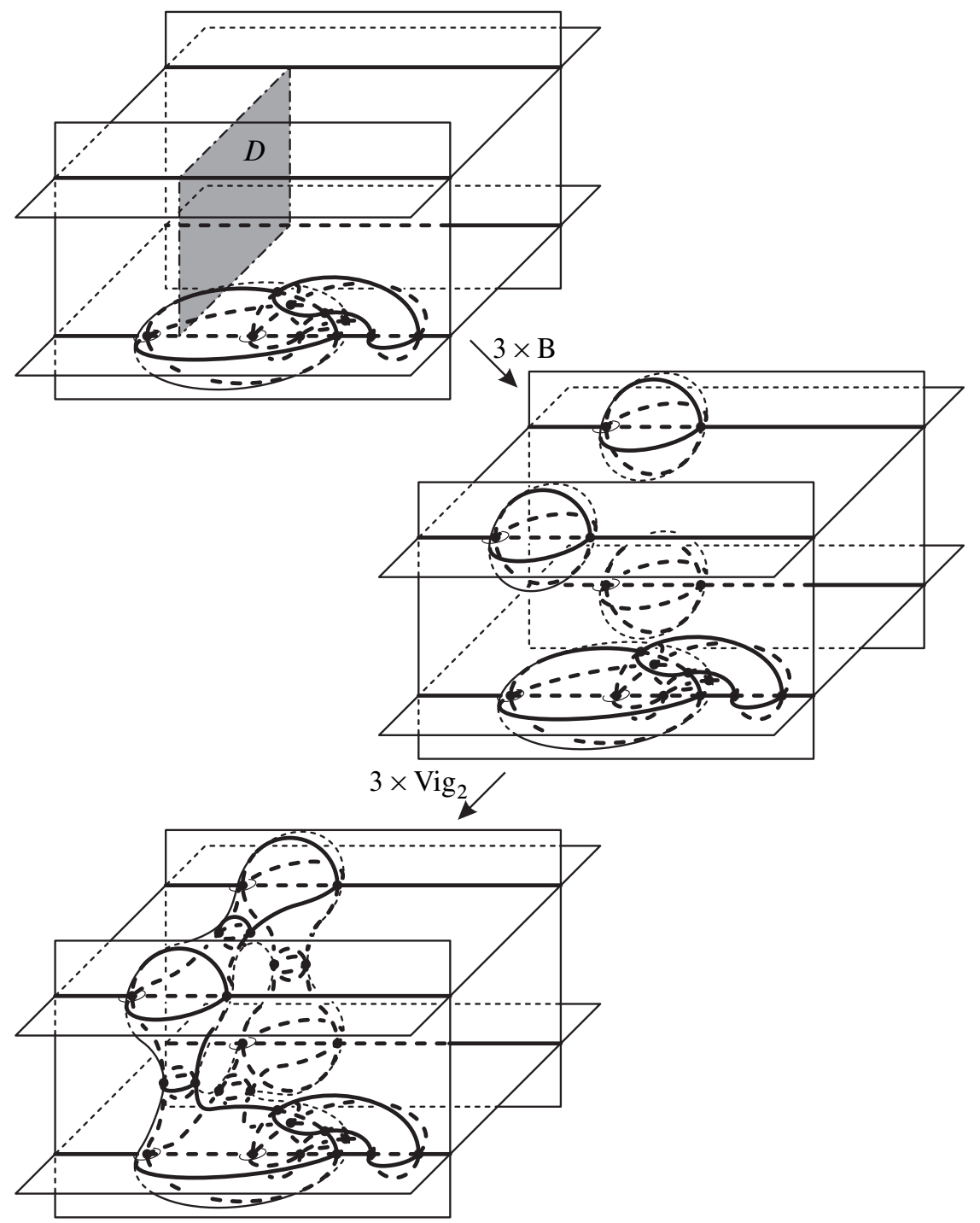

Figure 28: Preparation before moving the small wall (second part)

$\mathrm{T}_{*}$-moves to put the wall in the right position; the result is shown in Figure 33. Note that there are many possibilities for accomplishing this task; for instance, one can apply

- a positive and a negative $\mathrm{T}_{3}-$ move, twice,

- a positive $T_{1}-$ and a negative $T_{3}-$ move, twice,

- a positive $\mathrm{T}_{3}-$ and a negative $\mathrm{T}_{1}-$ move, thrice. 


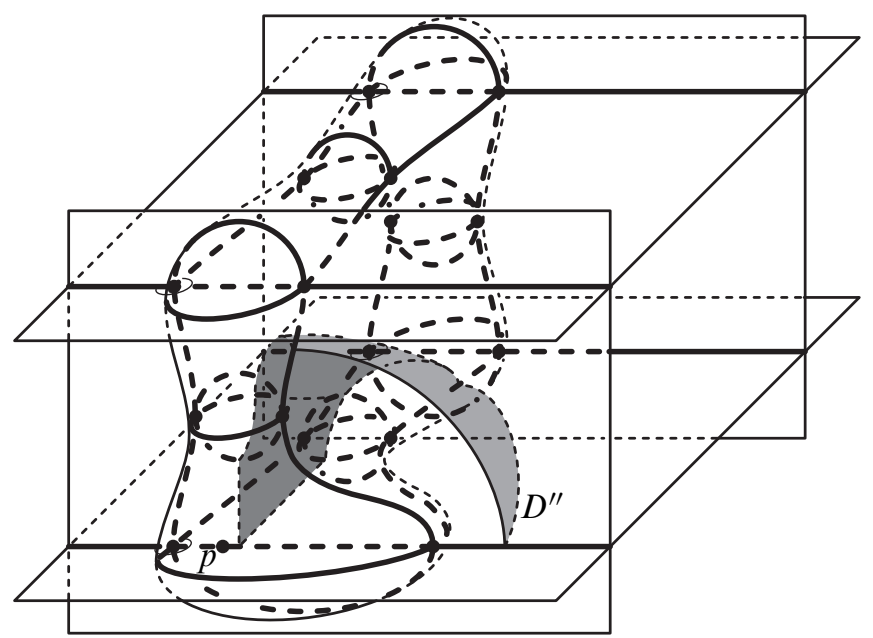

Figure 29: Preparation before moving the small wall (third part)

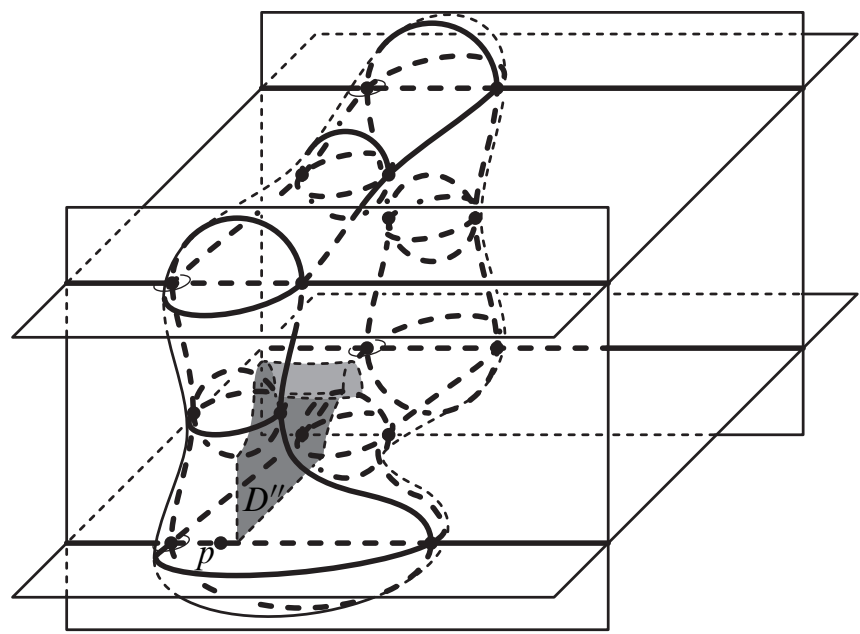

Figure 30: Preparation before moving the small wall (fourth part)

(If we have $\#(\partial D \cap S(\Sigma)) \neq 4$, the situation is analogous; in fact, only the number of pairs of moves changes.) Finally, we apply the moves shown in Figures 27 and 28 in reverse order. The result is $\Sigma_{D, p}$, which has been obtained from $\Sigma$ via $\mathrm{T}_{*}-, \mathrm{B}-$ and $\mathrm{Vig}_{2}-$ moves. The proof is complete. 

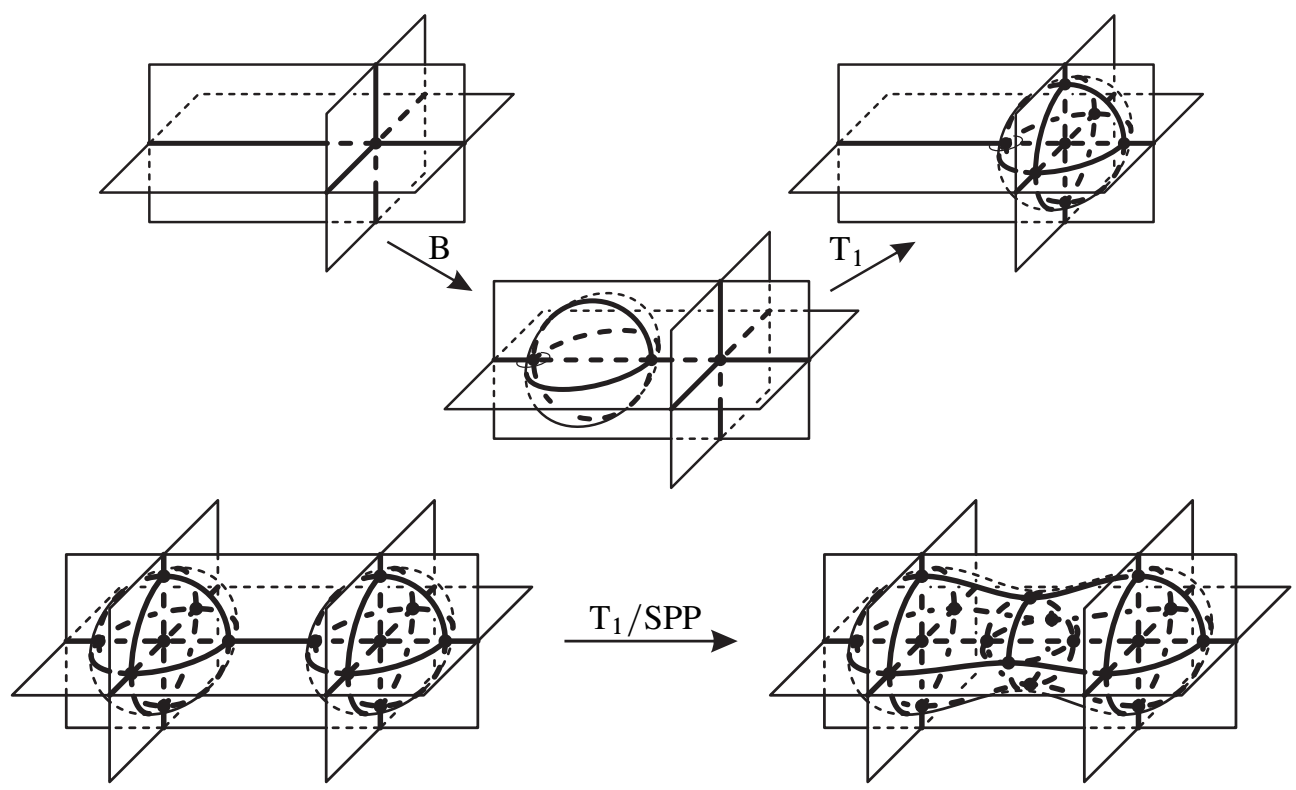

Figure 31: Moves to simplify the self-adjacency of the closure of $C^{\prime}$ near each triple point (above) and edge (below) of $\Sigma_{D^{\prime}, p}$ where we have a selfadjacency of the closure of $C^{\prime}$. (For the edge case, one of the triple points of each sphere constructed above is replaced by a spiral piping.)

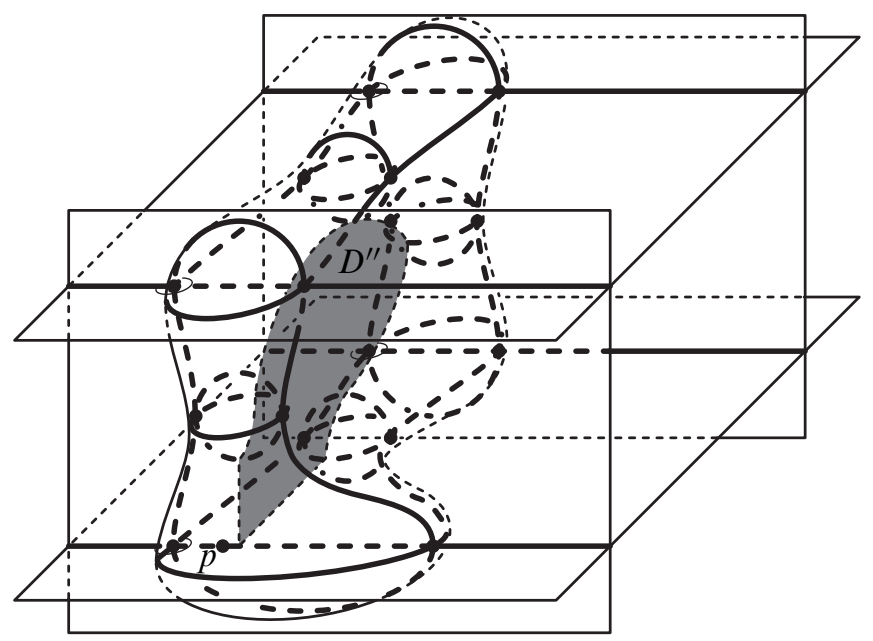

Figure 32: Configuration after moving the small wall through $C^{\prime}$ 


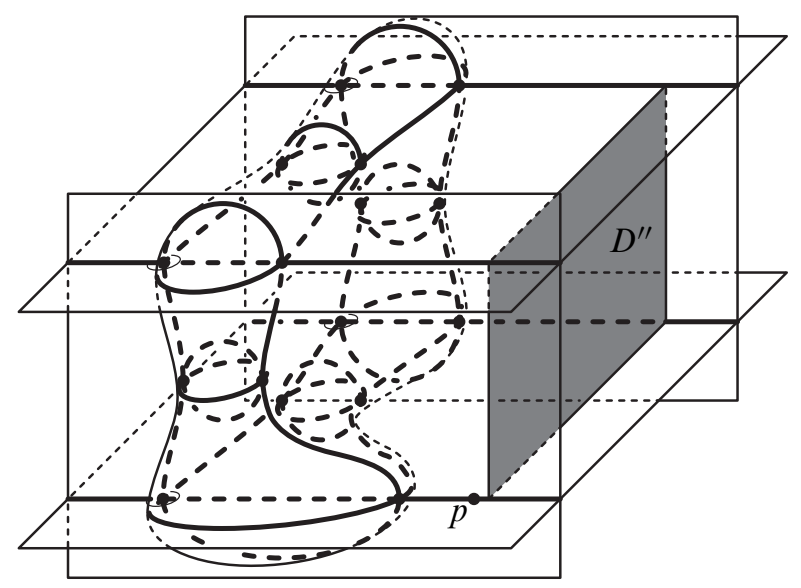

Figure 33: The wall is in the right position

\section{The state-sum invariant}

Theorem 5 allows us to define a state-sum invariant of closed 3-manifolds.

Let $\Sigma$ be a nullhomotopic filling Dehn sphere of a closed 3-manifold $M$. Recall that $T(\Sigma)$ is the set of triple points of $\Sigma$ and that $\Sigma \backslash S(\Sigma)$ (ie the set of simple points) consists of disjoint discs. Let us call $C(\Sigma)$ the class of these discs.

Moreover, let $\mathcal{F}$ be a finite set consisting of $m>1$ elements (called colours). An $\mathcal{F}-$ colouring of $\Sigma$ is a map $\varphi: C(\Sigma) \rightarrow \mathcal{F}$. The set of all $\mathcal{F}$-colourings of $\Sigma$ is denoted by $\Phi_{\mathcal{F}}(\Sigma)$. If $\widetilde{C} \subset C(\Sigma)$, we denote by $\Phi_{\mathcal{F}}(\widetilde{C})$ the set of maps $\varphi: \widetilde{C} \rightarrow \mathcal{F}$. Note that $\Phi_{\mathcal{F}}(\Sigma)$ can be identified with $\Phi_{\mathcal{F}}(C(\Sigma) \backslash \widetilde{C}) \times \Phi_{\mathcal{F}}(\widetilde{C})$. If $\varphi$ is an $\mathcal{F}$-colouring of $\Sigma$, we can associate a symbol

$$
p^{\varphi}:=\left|\begin{array}{lll}
a_{1} & b_{1} & c_{1} \\
a_{2} & b_{2} & c_{2} \\
a_{3} & b_{3} & c_{3} \\
a_{4} & b_{4} & c_{4}
\end{array}\right|
$$

to each $p \in T(\Sigma)$, where the $a_{*}$ 's, the $b_{*}$ 's and the $c_{*}$ 's are shown in Figure 34 . Since this definition involves some choices about the identification of the neighbourhood of $p$ with the abstract picture above, we assume that each symbol is invariant under a change of this identification. More precisely, we assume that the identities

$$
\left|\begin{array}{lll}
a_{1} & b_{1} & c_{1} \\
a_{2} & b_{2} & c_{2} \\
a_{3} & b_{3} & c_{3} \\
a_{4} & b_{4} & c_{4}
\end{array}\right|=\left|\begin{array}{lll}
c_{1} & a_{1} & b_{1} \\
c_{2} & a_{2} & b_{2} \\
c_{3} & a_{3} & b_{3} \\
c_{4} & a_{4} & b_{4}
\end{array}\right|=\left|\begin{array}{lll}
a_{4} & c_{1} & b_{2} \\
a_{1} & c_{4} & b_{3} \\
a_{2} & c_{3} & b_{4} \\
a_{3} & c_{2} & b_{1}
\end{array}\right|=\left|\begin{array}{lll}
a_{1} & b_{4} & c_{2} \\
a_{2} & b_{3} & c_{1} \\
a_{3} & b_{2} & c_{4} \\
a_{4} & b_{1} & c_{3}
\end{array}\right|
$$




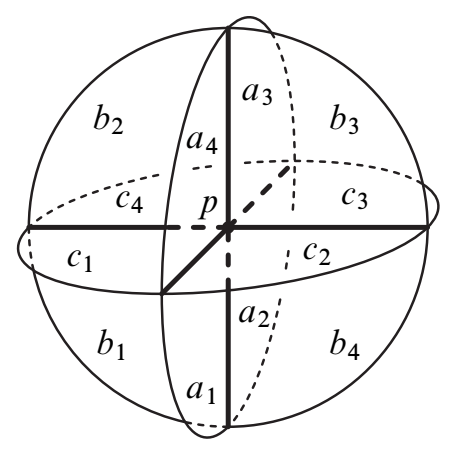

Figure 34: Colours near a triple point $p$

hold for all $a_{*}, b_{*}, c_{*} \in \mathcal{F}$. To be precise, we should distinguish a symbol from its equivalence class; nevertheless, to avoid proliferation of notation, we use the same notation for both the symbol and its equivalence class.

Let us consider now the polynomial ring $\mathcal{R}:=\mathbb{F}\left[s_{1}, \ldots, s_{N}\right]$, where $\mathbb{F}$ is a field and the $s_{*}$ 's are (the equivalence classes of) the symbols. The polynomial

$$
S S_{m}(\Sigma):=\sum_{\varphi \in \Phi_{\mathcal{F}}(\Sigma)}\left(\prod_{p \in T(\Sigma)} p^{\varphi}\right)
$$

of $\mathcal{R}$ is called state sum of $\Sigma$ of type $m$. Note that $S S_{m}(\Sigma)$ is an invariant of $\Sigma$, but it is not an invariant of $M$; in fact, it depends on the particular nullhomotopic filling Dehn sphere $\Sigma$ of $M$.

By virtue of Theorem 5, any nullhomotopic filling Dehn sphere $\Sigma^{\prime}$ of $M$ can be obtained from $\Sigma$ via $\mathrm{T}_{*}-, \mathrm{B}-$ and $\mathrm{S}-$ moves. Since the moves are local, one can compute the difference $S S_{m}(\Sigma)-S S_{m}\left(\Sigma^{\prime}\right)$, and prove that it belongs to the ideal $I_{m}$ generated by the following polynomials:

$$
\begin{gathered}
\left|\begin{array}{lll}
\alpha_{6} & a_{2} & A_{1} \\
\alpha_{3} & a_{3} & A_{2} \\
\alpha_{4} & a_{4} & A_{3} \\
\alpha_{5} & a_{5} & A_{4}
\end{array}\right|\left|\begin{array}{lll}
\alpha_{1} & a_{1} & B_{1} \\
\alpha_{2} & a_{2} & B_{2} \\
\alpha_{3} & a_{5} & B_{3} \\
\alpha_{6} & a_{6} & B_{4}
\end{array}\right|- \\
\sum_{X_{\star}, Y_{\star}, z_{\star}, \zeta_{\star} \in \mathcal{F}}\left|\begin{array}{lll}
\alpha_{1} & a_{1} & X_{1} \\
\alpha_{2} & z_{1} & X_{2} \\
\zeta_{2} & z_{2} & X_{3} \\
\zeta_{1} & a_{6} & X_{4}
\end{array}\right|\left|\begin{array}{lll}
\zeta_{1} & z_{1} & Y_{1} \\
\zeta_{2} & a_{3} & Y_{2} \\
\alpha_{4} & a_{4} & Y_{3} \\
\alpha_{5} & z_{2} & Y_{4}
\end{array}\right|\left|\begin{array}{ccc}
\alpha_{6} & B_{1} & A_{1} \\
\alpha_{1} & Y_{1} & A_{2} \\
\zeta_{1} & Y_{2} & X_{2} \\
\alpha_{5} & B_{2} & X_{1}
\end{array}\right|\left|\begin{array}{lll}
B_{2} & a_{6} & X_{2} \\
B_{3} & z_{2} & A_{2} \\
Y_{3} & a_{4} & A_{3} \\
Y_{2} & a_{5} & X_{3}
\end{array}\right|\left|\begin{array}{lll}
\alpha_{2} & B_{4} & X_{4} \\
\alpha_{3} & Y_{4} & X_{3} \\
\alpha_{4} & Y_{3} & A_{3} \\
\zeta_{2} & B_{3} & A_{4}
\end{array}\right|\left|\begin{array}{lll}
B_{1} & a_{2} & A_{1} \\
B_{4} & a_{3} & X_{1} \\
Y_{4} & z_{1} & X_{4} \\
Y_{1} & a_{1} & A_{4}
\end{array}\right|,
\end{gathered}
$$




$$
\begin{gathered}
\sum_{x_{\star} \in \mathcal{F}}\left|\begin{array}{lll}
\alpha_{8} & x_{1} & A_{2} \\
\alpha_{3} & x_{2} & A_{3} \\
\alpha_{4} & a_{4} & A_{4} \\
\alpha_{7} & a_{5} & A_{5}
\end{array}\right|\left|\begin{array}{lll}
\alpha_{1} & a_{1} & \beta_{8} \\
\alpha_{2} & x_{1} & \beta_{1} \\
\alpha_{3} & a_{5} & \beta_{2} \\
\alpha_{8} & a_{6} & \beta_{3}
\end{array}\right|\left|\begin{array}{ccc}
\beta_{8} & a_{1} & A_{1} \\
\beta_{3} & a_{2} & A_{2} \\
\beta_{4} & x_{2} & A_{5} \\
\beta_{7} & x_{1} & A_{6}
\end{array}\right|\left|\begin{array}{lll}
\alpha_{7} & x_{2} & \beta_{7} \\
\alpha_{4} & a_{2} & \beta_{6} \\
\alpha_{5} & a_{3} & \beta_{5} \\
\alpha_{6} & a_{4} & \beta_{4}
\end{array}\right|- \\
\sum_{Y_{\star} \in \mathcal{F}}\left|\begin{array}{lll}
\alpha_{1} & a_{1} & A_{1} \\
\alpha_{2} & a_{2} & Y_{1} \\
\alpha_{5} & a_{3} & Y_{2} \\
\alpha_{6} & a_{6} & A_{6}
\end{array}\right| \begin{array}{lll}
\alpha_{8} & \beta_{8} & A_{2} \\
\alpha_{1} & \beta_{7} & A_{3} \\
\alpha_{6} & \beta_{6} & Y_{1} \\
\alpha_{7} & \beta_{1} & A_{1}
\end{array}|| \begin{array}{lll}
\beta_{1} & a_{6} & Y_{1} \\
\beta_{2} & a_{3} & A_{3} \\
\beta_{5} & a_{4} & A_{4} \\
\beta_{6} & a_{5} & Y_{2}
\end{array}|| \begin{array}{lll}
\alpha_{2} & \beta_{3} & A_{6} \\
\alpha_{3} & \beta_{4} & Y_{2} \\
\alpha_{4} & \beta_{5} & A_{4} \\
\alpha_{5} & \beta_{2} & A_{5}
\end{array} \mid,
\end{gathered}
$$$$
\sum_{x \in \mathcal{F}}\left|\begin{array}{ccc}
\alpha_{6} & x & A_{2} \\
\alpha_{3} & a_{3} & A_{3} \\
\alpha_{4} & a_{4} & A_{4} \\
\alpha_{5} & a_{5} & A_{5}
\end{array}\right|\left|\begin{array}{ccc}
\alpha_{1} & a_{1} & \beta_{6} \\
\alpha_{2} & x & \beta_{1} \\
\alpha_{3} & a_{5} & \beta_{2} \\
\alpha_{6} & a_{6} & \beta_{3}
\end{array}\right|\left|\begin{array}{ccc}
\beta_{6} & a_{1} & A_{1} \\
\beta_{3} & a_{2} & A_{2} \\
\beta_{4} & a_{3} & A_{5} \\
\beta_{5} & x & A_{6}
\end{array}\right|-
$$$$
\sum_{Y_{\star}, v, \mu_{\star}, \zeta_{\star} \in \mathcal{F}}\left|\begin{array}{ccc}
\alpha_{1} & a_{1} & A_{1} \\
\alpha_{2} & a_{2} & Y_{1} \\
\zeta_{2} & v & Y_{2} \\
\zeta_{1} & a_{6} & A_{6}
\end{array}\right|\left|\begin{array}{ccc}
\zeta_{1} & a_{2} & \beta_{5} \\
\zeta_{2} & a_{3} & \mu_{1} \\
\alpha_{4} & a_{4} & \mu_{2} \\
\alpha_{5} & v & \beta_{4}
\end{array}\right|\left|\begin{array}{ccc}
\alpha_{6} & \beta_{6} & A_{2} \\
\alpha_{1} & \beta_{5} & A_{3} \\
\zeta_{1} & \mu_{1} & Y_{1} \\
\alpha_{5} & \beta_{1} & A_{1}
\end{array}\right|\left|\begin{array}{ccc}
\beta_{1} & a_{6} & Y_{1} \\
\beta_{2} & v & A_{3} \\
\mu_{2} & a_{4} & A_{4} \\
\mu_{1} & a_{5} & Y_{2}
\end{array}\right|\left|\begin{array}{ccc}
\alpha_{2} & \beta_{3} & A_{6} \\
\alpha_{3} & \beta_{4} & Y_{2} \\
\alpha_{4} & \mu_{2} & A_{4} \\
\zeta_{2} & \beta_{2} & A_{5}
\end{array}\right|
$$

$$
\begin{aligned}
\sum_{x, Y, \mu, \zeta \in \mathcal{F}}\left|\begin{array}{ccc}
\alpha_{6} & x & A_{2} \\
\zeta & a_{3} & A_{3} \\
\alpha_{4} & a_{4} & A_{4} \\
\alpha_{5} & a_{5} & Y
\end{array}\right|\left|\begin{array}{ccc}
\alpha_{1} & a_{1} & \beta_{6} \\
\alpha_{2} & x & \beta_{1} \\
\zeta & a_{5} & \beta_{2} \\
\alpha_{6} & a_{6} & \mu
\end{array}\right|\left|\begin{array}{ccc}
\beta_{6} & a_{1} & A_{1} \\
\mu & a_{2} & A_{2} \\
\beta_{4} & a_{3} & Y \\
\beta_{5} & x & A_{6}
\end{array}\right|\left|\begin{array}{ccc}
\zeta & \mu & Y \\
\alpha_{2} & \beta_{4} & A_{4} \\
\alpha_{3} & \beta_{3} & A_{5} \\
\alpha_{4} & \beta_{2} & A_{6}
\end{array}\right|- \\
\sum_{x^{\prime}, Y^{\prime}, \mu^{\prime}, \zeta^{\prime} \in \mathcal{F}}\left|\begin{array}{ccc}
\alpha_{1} & a_{1} & A_{1} \\
\alpha_{2} & a_{2} & Y^{\prime} \\
\alpha_{3} & x^{\prime} & A_{5} \\
\zeta^{\prime} & a_{6} & A_{6}
\end{array}\right|\left|\begin{array}{ccc}
\zeta^{\prime} & a_{2} & \beta_{5} \\
\alpha_{3} & a_{3} & \mu^{\prime} \\
\alpha_{4} & a_{4} & \beta_{3} \\
\alpha_{5} & x^{\prime} & \beta_{4}
\end{array}\right|\left|\begin{array}{ccc}
\alpha_{6} & \beta_{6} & A_{2} \\
\alpha_{1} & \beta_{5} & A_{3} \\
\zeta^{\prime} & \mu^{\prime} & Y^{\prime} \\
\alpha_{5} & \beta_{1} & A_{1}
\end{array}\right|\left|\begin{array}{lll}
\beta_{1} & a_{6} & Y^{\prime} \\
\beta_{2} & x^{\prime} & A_{3} \\
\beta_{3} & a_{4} & A_{4} \\
\mu^{\prime} & a_{5} & A_{5}
\end{array}\right|,
\end{aligned}
$$$$
\sum_{v_{\star}, x_{\star}, Y_{\star}, \mu_{\star}, \zeta_{\star} \in \mathcal{F}}\left|\begin{array}{lll}
\zeta_{1} & a_{1} & A_{1} \\
\zeta_{2} & a_{2} & Y_{1} \\
\zeta_{3} & x_{2} & Y_{6} \\
\zeta_{4} & x_{1} & A_{2}
\end{array}\right|\left|\begin{array}{ccc}
\zeta_{1} & x_{1} & Y_{1} \\
\mu_{1} & x_{2} & A_{1} \\
\mu_{2} & x_{3} & Y_{5} \\
\zeta_{4} & x_{4} & Y_{6}
\end{array}\right|\left|\begin{array}{ccc}
\zeta_{1} & x_{4} & A_{1} \\
\mu_{1} & x_{3} & Y_{2} \\
\mu_{2} & x_{2} & Y_{4} \\
\zeta_{4} & x_{1} & Y_{5}
\end{array}\right|\left|\begin{array}{lll}
\zeta_{1} & x_{1} & Y_{2} \\
\zeta_{2} & x_{2} & A_{1} \\
\zeta_{3} & a_{2} & Y_{3} \\
\zeta_{4} & a_{1} & Y_{4}
\end{array}\right|\left|\begin{array}{lll}
\mu_{1} & v_{1} & Y_{5} \\
a_{1} & v_{2} & Y_{4} \\
a_{2} & \zeta_{3} & Y_{3} \\
\mu_{2} & \zeta_{2} & A_{1}
\end{array}\right|\left|\begin{array}{lll}
\mu_{1} & \zeta_{2} & Y_{6} \\
a_{1} & \zeta_{3} & Y_{5} \\
a_{2} & v_{2} & A_{1} \\
\mu_{2} & v_{1} & A_{2}
\end{array}\right|,
$$$$
\sum_{x, Y \in \mathcal{F}}\left|\begin{array}{ccc}
\alpha_{1} & a_{1} & A_{1} \\
\alpha_{2} & a_{2} & A_{2} \\
\alpha_{6} & x & Y \\
\alpha_{5} & a_{6} & A_{6}
\end{array}\right|\left|\begin{array}{ccc}
\alpha_{2} & a_{3} & A_{6} \\
\alpha_{3} & a_{2} & Y \\
\alpha_{5} & x & A_{2} \\
\alpha_{6} & a_{6} & A_{5}
\end{array}\right|\left|\begin{array}{ccc}
\alpha_{3} & a_{3} & A_{5} \\
\alpha_{2} & a_{4} & A_{2} \\
\alpha_{4} & a_{5} & A_{3} \\
\alpha_{5} & a_{6} & A_{4}
\end{array}\right|-\sum_{X, y \in \mathcal{F}}\left|\begin{array}{ccc}
\alpha_{1} & a_{1} & A_{1} \\
\alpha_{2} & a_{2} & A_{2} \\
\alpha_{6} & a_{5} & A_{3} \\
\alpha_{5} & a_{6} & A_{6}
\end{array}\right|\left|\begin{array}{ccc}
\alpha_{2} & a_{3} & A_{6} \\
\alpha_{3} & a_{2} & A_{3} \\
\alpha_{5} & a_{5} & X \\
\alpha_{6} & y & A_{5}
\end{array}\right|\left|\begin{array}{ccc}
\alpha_{3} & a_{3} & A_{5} \\
\alpha_{2} & a_{4} & X \\
\alpha_{4} & a_{5} & A_{3} \\
\alpha_{5} & y & A_{4}
\end{array}\right|,
$$

for all $a_{1}, \ldots, a_{6}, A_{1}, \ldots, A_{6}, B_{1}, \ldots, B_{4}, \alpha_{1}, \ldots, \alpha_{8}, \beta_{1}, \ldots, \beta_{8} \in \mathcal{F}$. Therefore, we obtain the following. 
Corollary 9 The coset

$$
\operatorname{inv}_{m}(M)=S S_{m}(\Sigma)+I_{m} \in \mathcal{R} / I_{m}
$$

does not depend on the particular nullhomotopic filling Dehn sphere $\Sigma$ presenting the closed 3-manifold $M$, and thus it is an invariant of $M$.

Other invariants From the invariant $\operatorname{inv}_{m}$, a number of other invariants can be constructed (see King [9] for the Turaev-Viro version of these modifications): for instance,

- by colouring also the edges of the nullhomotopic filling Dehn sphere $\Sigma$,

- by giving weights to the regions of $\Sigma$,

- by assuming that some simplifying identities hold,

- by considering the coset with respect to the radical $\sqrt{I_{m}}$ of $I_{m}$ or with respect to any other ideal $I$ containing $I_{m}$.

Computation of the invariant After the definition of the invariant $\operatorname{inv}_{m}(M)$, the issue of computing it naturally arises. We have not made any computation as yet, but our plan is to use two techniques to compute $\operatorname{inv}_{m}(M)$.

- If we find an element of the zero variety associated to $I_{m}$, we can evaluate the state sum $S S_{m}(\Sigma)$ at it, getting an element of $\mathbb{F}$ which is obviously an invariant of $M$.

- If we find a Gröbner basis of $\mathcal{R} / I_{m}$, we can find the normal form of $\operatorname{inv}_{m}(M)$ and hence check whether two closed 3-manifolds share the same invariant or not.

Relationship with the Turaev-Viro invariant The framework we have used to define the invariant $\operatorname{inv}_{m}$ is analogous to that used to define the Turaev-Viro invariant [16]. However, the two calculuses (and their proofs) used in the definition of the invariants are different. Hence, the following question naturally arises.

Question 10 Are the invariant $\operatorname{inv}_{m}$ and the Turaev-Viro invariant related (in some sense) to each other? 
Lower bounds for the Matveev complexity We conclude with a potential application of the invariant $\operatorname{inv}_{m}$.

A 3-manifold is $\mathbb{P}^{2}$-irreducible if every sphere embedded in it bounds a ball and every projective plane embedded in it (if any) is one-sided. Let $\mathcal{M}$ be the class of $\mathbb{P}^{2}$-irreducible closed 3-manifolds different from the 3-sphere $S^{3}$, the projective space $\mathbb{R} \mathbb{P}^{3}$ or the lens space $L(3,1)$. The Matveev complexity is defined for any compact 3-manifold using spines [10]. However, as shown in [11], if the 3-manifold $M$ is $\mathbb{P}^{2}$-irreducible and closed, the Matveev complexity $c(M)$ can be defined to be

- zero, if $M$ is $S^{3}, \mathbb{R P}^{3}$ or $L(3,1)$,

- the minimal number of tetrahedra among all one-vertex triangulations of $M$, if $M \in \mathcal{M}$.

It is quite easy to find good estimates for the Matveev complexity, but an exact calculation of it is very difficult. For instance, precise upper bounds can be easily found by exhibiting suitable triangulations, but lower bounds are usually rough. Here "precise" means that the Matveev complexity is a posteriori usually very close (if not equal) to the upper bound.

By using nullhomotopic filling Dehn spheres, a first lower bound for the Matveev complexity can be easily found. Let us denote by $\operatorname{cs}(M)$ the minimal number of triple points among all nullhomotopic filling Dehn spheres of $M$ (in [19] the invariant $\operatorname{cs}(M)$ is called nullhomotopic genus 0 triple point number). By means of the construction of nullhomotopic filling Dehn spheres made in the proof of Proposition 2 (see Figure 3), we obtain the inequality $c(M) \geqslant \operatorname{cs}(M) / 4$ for each $M \in \mathcal{M}$.

In practice, this result seems to be useless for finding a lower bound for the Matveev complexity directly. We describe a potential application of the invariant $\operatorname{inv}_{m}$ to overcome this problem. If $P$ is a polynomial in $\mathcal{R}$, let $\operatorname{deg}(P)$ be its total degree. Moreover, for any subset $\mathcal{P} \subset \mathcal{R}$, let $\operatorname{deg}(\mathcal{P})=\min \{\operatorname{deg}(P): P \in \mathcal{P}\}$. For each $m>1$, the inequality $\operatorname{cs}(M) \geqslant \operatorname{deg}\left(\operatorname{inv}_{m}(M)\right)$ holds, and hence the lower bound $c(M) \geqslant \operatorname{deg}\left(\operatorname{inv}_{m}(M)\right) / 4$ holds for each $M \in \mathcal{M}$.

It is worth noting that such a framework can be equally applied to the Turaev-Viro setting, leading to non-sharp lower bounds on complexity, as shown by King [9]. Hence, the following question naturally arises.

Question 11 Are the lower bounds obtained via $\operatorname{inv}_{m}$ sharp, at least for some closed 3-manifolds? 
Acknowledgements I am grateful to Professor Sergei Matveev and Simon King for useful discussions during the beautiful period I spent at the Department of Mathematics in Darmstadt. I would like to thank the Galileo Galilei Doctoral School of Pisa and the DAAD (Deutscher Akademischer Austausch Dienst) for giving me the opportunity to reside in Darmstadt, and Professor Alexander Martin for his assistance. I would also like to thank the referee for his or her useful comments and corrections.

This paper is dedicated to Paolo.

\section{References}

[1] I R Aitchison, S Matsumoto, J H Rubinstein, Immersed surfaces in cubed manifolds, Asian J. Math. 1 (1997) 85-95 MR1480991

[2] E K Babson, C S Chan, Counting faces of cubical spheres modulo two, from: "Combinatorics and applications (Tianjin, 1996)", (W Y C Chen, D-Z Du, F D Hsu, H P Yap, editors), Discrete Math. 212 (2000) 169-183 MR1748648

[3] B G Casler, An imbedding theorem for connected 3-manifolds with boundary, Proc. Amer. Math. Soc. 16 (1965) 559-566 MR0178473

[4] N P Dolbilin, M A Shtan' ko, M I Shtogrin, Cubic manifolds in lattices, Izv. Ross. Akad. Nauk Ser. Mat. 58 (1994) 93-107 MR1275903

[5] L Funar, Cubulations, immersions, mappability and a problem of Habegger, Ann. Sci. École Norm. Sup. (4) 32 (1999) 681-700 MR1710756

[6] J Hass, J Hughes, Immersions of surfaces in 3-manifolds, Topology 24 (1985) 97-112 MR790679

[7] T Homma, T Nagase, On elementary deformations of maps of surfaces into 3manifolds. I, Yokohama Math. J. 33 (1985) 103-119 MR817976

[8] T Homma, T Nagase, On elementary deformations of maps of surfaces into 3manifolds. II, from: "Topology and computer science (Atami, 1986)", (S Suzuki, editor), Kinokuniya, Tokyo (1987) 1-20 MR1112583

[9] S A King, Ideal Turaev-Viro invariants, Topology Appl. 154 (2007) 1141-1156 MR2298630

[10] S V Matveev, The theory of the complexity of three-dimensional manifolds, Akad. Nauk Ukrain. SSR Inst. Mat. Preprint 13 (1988) MR982986

[11] S V Matveev, Algorithmic topology and classification of 3-manifolds, Algorithms and Computation in Math. 9, Springer, Berlin (2003) MR1997069

[12] J M Montesinos-Amilibia, Representing 3-manifolds by Dehn spheres, from: "Mathematical contributions: volume in honor of Professor Joaquín Arregui Fernández (Spanish)", (J L López, L M P Coronado, editors), Homen. Univ. Complut., Editorial Complutense, Madrid (2000) 239-247 MR1803906 
[13] C D Papakyriakopoulos, On Dehn's lemma and the asphericity of knots, Ann. of Math. (2) 66 (1957) 1-26 MR0090053

[14] D Roseman, Reidemeister-type moves for surfaces in four-dimensional space, from: "Knot theory (Warsaw, 1995)", (V F R Jones, J Kania-Bartoszyńska, J H Przytycki, P Traczyk, V G Turaev, editors), Banach Center Publ. 42, Polish Acad. Sci., Warsaw (1998) 347-380 MR1634466

[15] M A Shtan' ko, M I Shtogrin, Embedding cubic manifolds and complexes into a cubic lattice, Uspekhi Mat. Nauk 47 (1992) 219-220 MR1171874

[16] V G Turaev, O Y Viro, State sum invariants of 3-manifolds and quantum $6 j$-symbols, Topology 31 (1992) 865-902 MR1191386

[17] R Vigara, A new proof of a theorem of J M Montesinos, J. Math. Sci. Univ. Tokyo 11 (2004) 325-351 MR2097529

[18] R Vigara, Representación de 3-variedades por esferas de Dehn rellenantes, $\mathrm{PhD}$ thesis, UNED Madrid (2006)

[19] R Vigara, A set of moves for Johansson representation of 3-manifolds, Fund. Math. 190 (2006) 245-288 MR2232862

Department of Mathematics, University of Salento

Palazzo Fiorini, Via per Arnesano, I-73100 Lecce, Italy

amendola@mail.dm.unipi.it

http://www.dm.unipi.it/ amendola/

Received: 29 January 2008 Revised: 31 October 2008 